\title{
A rapeseed WRKY transcription factor phosphorylated by CPK modulates cell death and leaf senescence by regulating the expression of ROS and SA-synthesis-related genes
}

Xing Cui ${ }^{1}$, Peiyu Zhao ${ }^{1}$, Wanwan Liang ${ }^{1}$, Qian Cheng ${ }^{1}$, Bangbang Mu ${ }^{1}$, Fangfang Niu ${ }^{1}$, Jingli Yan ${ }^{1}$, Chunlin Liu' ${ }^{2}$ Hua Xie ${ }^{3}$, Nat N. V. Kav ${ }^{4}$, Michael K. Deyholos ${ }^{5}$, Yuan-Qing Jiang ${ }^{*}$, Bo Yang ${ }^{{ }^{*}}$

1. State Key Laboratory of Crop Stress Biology in Arid Areas, College of Life Sciences, Northwest A \& F University, Yangling, Shaanxi, 712100, China

2. Hunan State Key Laboratory of Crop Germplasm Innovation and Resource Utilization, Hunan Agricultural University, Changsha, China

3. Agrobiotechnology Research Center, Beijing Academy of Agricultural and Forestry Sciences, Beijing, China

4. Department of Agricultural, Food and Nutritional Science, University of Alberta, Edmonton, AB T6G 2P5, Canada

5. Department of Biology, University of British Columbia, Okanagan Campus, Kelowna, BC, V1V 1V7,

Canada

*Correspondence (Email: jiangyq@nwafu.edu.cn, yangwl@nwafu.edu.cn; Fax: 86-29-87092262; Tel: 86-29-

87092262 


\section{Supplementary data}

Table S1 Primers used in this study

Fig. S1 The coding region and translated sequence of BnaWSR1.

Fig. S2 Analysis of expression of two senescence marker genes during leaf development.

Fig. S3 Subcellular localization of BnaWSR1.

Fig. S4 Expression of BnaWSR1 in response to various treatments.

Fig. S5 Alignment of DNA binding domains of BnaWSR1 and representative AtWRKYs.

Fig. S6 Transcriptional activity assay of BnaWSR1.

Fig. S7 Negative controls of the BiFC assay.

Fig. S8 Examination of BnaWSR1 function in transient expression assay in tobacco.

Fig. S9 Expression of BnaWSR1ca in rapeseed protoplasts resulted in ROS accumulation and cell death.

Fig. S10 qRT-PCR analysis of BnaWSR1 level in constitutive overexpression plants.

Fig. S11 Expression analysis of BnaWSR1 in XVE-BnaWSR1ca transgenic lines through qRT-PCR.

Fig. S12 Expression analysis of SA and ROS production associated marker genes and senescence-related genes.

Fig. S13 Regulatory effect of BnaWSR1ca on the promoters of rapeseed genes.

Fig. S14 Inspection of purified GST-BnaWRS1 protein via SDS-PAGE.

Fig. S15 EMSA assay of binding of phosphomimic BnaWSR1 to the W-box.

Fig. S16 Sequence analysis of the promoter regions of putative targets of BnaWSR1.

Fig. S17 The ChIP-qPCR assay of binding of BnaWSR1 to relevant promoters of rapeseed genes.

Fig. S18 A proposed model illustrating BnaWSR1 involved in ROS- and SA-mediated leaf senescence. 
Table S1. Primers used in this study

\begin{tabular}{|c|c|c|}
\hline Primer name & Primer sequence $\left(5^{\prime} \rightarrow 3^{\prime}\right)$ & Purpose \\
\hline BnaWSR1-SpeI-F & GGACTAGTATGTCCAATGAAACCAAAGA & $\mathrm{BiFC}$ \\
\hline BnaWSR1-NotI-F & CATGCCATGGTATCCAATGAAACCAAAGAT & $\begin{array}{l}\text { transient expression in } \\
\text { protoplasts }\end{array}$ \\
\hline $\begin{array}{l}\text { BnaWSR1-GW-SalI- } \\
\text { R }\end{array}$ & $\begin{array}{l}\text { GTCTCCACCTCCGGAGTCGACATGCTCTTGCTT } \\
\text { GAAGAAAAT }\end{array}$ & $\begin{array}{l}\mathrm{BiFC}, \mathrm{GST} \text { fusion and } \\
\text { inducible overexpression } \\
\text { in Arabidopsis, transient } \\
\text { expression in protoplasts }\end{array}$ \\
\hline BnaWSR1_NdeI_F & $\begin{array}{l}\text { GGAATTCCATATGTCCAATGAAACCAAAGATT } \\
\text { TCTACAAC }\end{array}$ & yeast assay \\
\hline $\begin{array}{l}\text { BnaWSR1_BamHI_ } \\
\text { R (stop) }\end{array}$ & $\begin{array}{l}\text { CGGGATCCTCAATGCTCTTGCTTGAAGAAAAT } \\
\text { G }\end{array}$ & $\begin{array}{l}\text { constitutive } \\
\text { overexpression in } \\
\text { Arabidopsis }\end{array}$ \\
\hline BnaWSR1_NcoI-F & CATGCCATGGTATCCAATGAAACCAAAGAT & $\begin{array}{l}\text { subcellular localization } \\
\text { assay, constitutive and } \\
\text { inducible overexpression } \\
\text { in Arabidopsis }\end{array}$ \\
\hline BnaWSR1-XhoI-F & CGCTCGAGATGTCCAATGAAACCAAAGA & inducible overexpression \\
\hline BnaWSR1-SpeI-R & GGACTAGTTCAATGCTCTTGCTTGAAGA & inducible overexpression \\
\hline BnaWSR1_ApaI-R & CGGGGCCCATGCTCTTGCTTGAAGAAAATG & $\begin{array}{l}\text { subcellular localization } \\
\text { assay }\end{array}$ \\
\hline BnaWSR1-qPCR-F & GCGGCGGCTGCTATGTATTCTGACT & qRT-PCR \\
\hline BnaWSR1-qPCR-R & GTATCCATAATCCAAAGCCCCСТCCAC & \\
\hline $\begin{array}{l}\text { BnaWSR1-225AA- } \\
\text { BamHI-R }\end{array}$ & CGGGATCCTCACGGAATGGGATGGTTGTGTT & $\begin{array}{l}\text { Deletion form D1 for } \\
\text { yeast assay }\end{array}$ \\
\hline $\begin{array}{l}\text { BnaWSR1- } \\
\text { 226AANcoI-F }\end{array}$ & CGCCATGGAGACTAATCTTCGGGGAAATTCC & $\begin{array}{l}\text { Deletion form D2 for } \\
\text { yeast assay }\end{array}$ \\
\hline $\begin{array}{l}\text { BraWSR1-promoter- } \\
\text { Sall-F }\end{array}$ & $\begin{array}{l}\text { CGCGTCGACATGGAATGATGTAGAATGGTGGA } \\
\text { TTCCATTAA }\end{array}$ & Promoter-GUS fusion \\
\hline $\begin{array}{l}\text { BraWSR1-promoter- } \\
\text { EcoRI-R }\end{array}$ & $\begin{array}{l}\text { CGGAATTCGGTGAAGAACAATGAAGAGATGAA } \\
\text { ACC }\end{array}$ & \\
\hline BnaWSR1-EcoRI-F & CGGAATTCATGTCCAATGAAACCAAAGAT & GST fusion \\
\hline $\begin{array}{l}\text { BnaWSR1- } \\
\text { T192D/T193D-F }\end{array}$ & $\begin{array}{l}\text { TACTATAGATGCGATGATCAAAAATGTAACGT } \\
\text { GAAGAAACGA }\end{array}$ & Site-directed mutagenesis \\
\hline BnaWSR1- & GTTACATTTTTGATCATCGCATCTATAGTAACT & \\
\hline T192D/T193D-R & CCTTGGG & \\
\hline & $\begin{array}{l}\text { TACTATAGATGCGCGGCACAAAAATGTAACGT } \\
\text { GAAGA }\end{array}$ & Site-directed mutagenesis \\
\hline $\begin{array}{l}\text { BnaWSR1- } \\
\text { T192AT193A-R }\end{array}$ & $\begin{array}{l}\text { GTTACATTTTTGTGCCGCGCATCTATAGTAACT } \\
\text { CCTTG }\end{array}$ & \\
\hline ProAtICS1-KpnI-F & GGGGTACCACTCAGACTCCAGAAGACGAC & Dual-LUC \\
\hline ProAtICS1-NcoI-R & $\begin{array}{l}\text { CGCCATGGCAGAAATTCGTAAAGTGTTTCTTGA } \\
\text { AGA }\end{array}$ & \\
\hline ProAtICS1-W4-F & TATTTCTAGTTATTTGACTAACCAAATCGA & EMSA \\
\hline ProAtICS1-W4-R & TCGATTTGGTTAGTCAAATAACTAGAAATA & \\
\hline ProAtICS1-W3-F & GATAAAATAACAATTTGACTTCTAAAGTCTAGT & EMSA \\
\hline ProAtICS1-W3-R & ACTAGACTTTAGAAGTCAAATTGTTATTTTATC & \\
\hline ProAtICS1-W2-F & AACAATATTCATTTTGACCAGGTTGAGTAA & EMSA \\
\hline ProAtICS1-W2-R & TTACTCAACCTGGTCAAAATGAATATTGTT & \\
\hline ProAtICS1-W1-F & CGTGTGATCTTAATTGACTCAAAATGAAAG & EMSA \\
\hline ProAtICS1-W1-F & CTTTCATTTTGAGTCAATTAAGATCACACG & \\
\hline
\end{tabular}




\begin{tabular}{|c|c|c|}
\hline ProAtICS1-mW3-F & $\begin{array}{l}\text { GATAAAATAACAATTTGGATTCTAAAGTCTAG } \\
\mathrm{T}\end{array}$ & EMSA \\
\hline ProAtICS1-mW3-R & ACTAGACTTTAGAATCCAAATTGTTATTTTATC & \\
\hline PAtICS1-ChIP-F0 & TCCATGCATTGCTCAAATTCATT & ChIP-qPCR \\
\hline PAtICS1-ChIP-R0 & TTCGTTTGGTTGATTTATCGTCG & \\
\hline ProAtICS1-ChIP-F2 & GGTTTCATTCTATTGGATTATCTGCA & ChIP-qPCR \\
\hline ProAtICS1-ChIP-R2 & ACCAAGTACATATTTGAGGAAACGA & \\
\hline ProAtICS1-ChIP-F3 & AGGTTATCTAAATTCAACTAACGTCC & ChIP-qPCR \\
\hline ProAtICS1-ChIP-R3 & AGTGTCGTATGCTTTAGTTCGAA & \\
\hline AtICS1-qPCR-F & ATGCGGGGACAGGGATAGTAG & qRT-PCR \\
\hline AtICS1-qPCR-R & TCGCCTGTAGAGATGTTGTTGC & \\
\hline AtICS2-qPCR-F & GTTTGCGGATGTCCAGTAGAAGAAGC & qRT-PCR \\
\hline AtICS2-qPCR-R & CTCCCCACCACCAAAGAACCCAATAG & \\
\hline ProAtPBS3-KpnI-F & GGGGTACCCAGGGCAACTAGAAGCAATCC & Dual-LUC \\
\hline $\begin{array}{l}\text { ProAtPBS3-BamHI- } \\
\text { R }\end{array}$ & $\begin{array}{l}\text { CGGGATCCATTTGGTTCTCTTTAAGTTGATATG } \\
\text { AAAAAATAA }\end{array}$ & \\
\hline ProAtPBS3-W1-F & ATACAGTTAAAACATTGACCCAGACTCACG & EMSA \\
\hline ProAtPBS3-W1-R & CGTGAGTCTGGGTCAATGTTTTAACTGTAT & \\
\hline ProAtPBS3-W2-F & TAGTTGCATAATGTTGACTATTAAATAATCG & EMSA \\
\hline ProAtPBS3-W2-R & CGATTATTTAATAGTCAACATTATGCAACTA & \\
\hline ProAtPBS3-mW2-F & TAGTTGCATAATGTTGAATATTAAATAATCG & EMSA \\
\hline ProAtPBS3-mW2-R & CGATTATTTAATATTCAACATTATGCAACTA & \\
\hline ProAtPBS3-W3-F & CTTAATATTTATATTTGACTCGCAGCGTAG & EMSA \\
\hline ProAtPBS3-W3-R & CTACGCTGCGAGTCAAATATAAATATTAAG & \\
\hline ProAtPBS3-W4-F & ATAAATAGTTTTTGTTGACTCCCATTGTAG & EMSA \\
\hline ProAtPBS3-W4-R & CTACAATGGGAGTCAACAAAAACTATTTAT & \\
\hline ProAtPBS3-ChIP-F1 & TCACACGCTAAGGTCAGTATTCT & ChIP-qPCR \\
\hline ProAtPBS3-ChIP-R1 & CCCTAACTAATTAAATGGATTTGGCA & \\
\hline ProAtPBS3-ChIP-F2 & ATTTCTCTGCGTTTTCCTGACTT & ChIP-qPCR \\
\hline ProAtPBS3-ChIP-R2 & CCTTGGGTTTTAACTTGTGACGA & \\
\hline ProAtPBS3-ChIP-F3 & ATCACTTTCATCACACGACTCAC & ChIP-qPCR \\
\hline ProAtPBS3-ChIP-R3 & CTCATACACAAAGACCAGACCAC & \\
\hline AtPBS3-qPCR-F & GAAGGAGACGGCACAGTTTGAGC & qRT-PCR \\
\hline AtPBS3-qPCR-R & GGCCCGATCGATCCGTCTTT & \\
\hline ProAtRbohD-KpnI-F & GGGGTACCGCGTCATGATCTTCCGTATGG & dual LUC \\
\hline ProAtRbohD-NcoI-R & $\begin{array}{l}\text { CGCCATGGAATTCGAGAAACCAAAAAGATCTC } \\
\text { ACTA }\end{array}$ & \\
\hline ProAtRbohD-W1-Sb & $\begin{array}{l}\text { AAAAAGTCAAAATAAATGGTCAGTAAAGGATT } \\
\text { TTGACCAGACC }\end{array}$ & EMSA \\
\hline $\begin{array}{l}\text { ProAtRbohD-W1- } \\
\text { ASb }\end{array}$ & $\begin{array}{l}\text { GGTCTGGTCAAAATCCTTTACTGACCATTTATT } \\
\text { TTGACTTTTT }\end{array}$ & \\
\hline ProAtRbohD-W2-Sb & AATGAATTATAGATTGACTTATGGAAAAGT & EMSA \\
\hline $\begin{array}{l}\text { ProAtRbohD-W2- } \\
\text { ASb }\end{array}$ & ACTTTTCCATAAGTCAATCTATAATTCATT & \\
\hline ProAtRbohD-W3-Sb & TGATCTAGCTAATTGACTTTCATATTTACA & EMSA \\
\hline
\end{tabular}




\begin{tabular}{|c|c|c|}
\hline $\begin{array}{l}\text { ProAtRbohD-W3- } \\
\text { ASb }\end{array}$ & TGTAAATATGAAAGTCAATTAGCTAGATCA & \\
\hline $\begin{array}{l}\text { ProAtRbohD-mW1- } \\
\text { S }\end{array}$ & $\begin{array}{l}\text { AAAAATCCAAAATAAATGGTCAGTAAAGGATT } \\
\text { TTGGACAGACC }\end{array}$ & EMSA \\
\hline $\begin{array}{l}\text { ProAtRbohD-mW1- } \\
\text { AS }\end{array}$ & $\begin{array}{l}\text { GGTCTGTCCAAAATCCTTTACTGACCATTTATT } \\
\text { TTGGATTTTT }\end{array}$ & \\
\hline $\begin{array}{l}\text { ProAtRbohD-mW2- } \\
\text { S }\end{array}$ & AATGAATTATAGATTGGATTATGGAAAAGT & EMSA \\
\hline $\begin{array}{l}\text { ProAtRbohD-mW2- } \\
\text { AS }\end{array}$ & ACTTTTCCATAATCCAATCTATAATTCATT & \\
\hline $\begin{array}{l}\text { ProAtRbohD-mW3- } \\
\text { S }\end{array}$ & TGATCTAGCTAATTGGATTTCATATTTACA & EMSA \\
\hline $\begin{array}{l}\text { ProAtRbohD-mW3- } \\
\text { AS }\end{array}$ & TGTAAATATGAAATCCAATTAGCTAGATCA & \\
\hline $\begin{array}{l}\text { ProAtRbohD-ChIP- } \\
\text { F1 }\end{array}$ & CACGGGGTTGGTGTATAAATGA & ChIP-qPCR \\
\hline $\begin{array}{l}\text { ProAtRbohD-ChIP- } \\
\text { R1 }\end{array}$ & TCAGGGAAGGTAGTGGGAAAC & \\
\hline $\begin{array}{l}\text { ProAtRbohD-ChIP- } \\
\text { F2 }\end{array}$ & ACAAGCCAAATGTTAGAAGCAAC & ChIP-qPCR \\
\hline $\begin{array}{l}\text { ProAtRbohD-ChIP- } \\
\text { R2 }\end{array}$ & CCCCGAGTTATGTCAAACACC & \\
\hline $\begin{array}{l}\text { ProAtRbohD-ChIP- } \\
\text { F3 }\end{array}$ & CGATGTTCCTTTAAGACTACCACA & ChIP-qPCR \\
\hline $\begin{array}{l}\text { ProAtRbohD-ChIP- } \\
\text { R3 }\end{array}$ & TCCATGTGAGATGTATTGTTCATTT & \\
\hline $\begin{array}{l}\text { ProAtRbohD-ChIP- } \\
\text { F4 }\end{array}$ & GCCGTAACGTGTGAATAGTGT & ChIP-qPCR \\
\hline $\begin{array}{l}\text { ProAtRbohD-ChIP- } \\
\text { R4 }\end{array}$ & CCGATGAGAAAGTAAGGTGGC & \\
\hline AtRbohD-qPCR-F & CCCCGGGAACGTGTTGTCTCTA & qRT-PCR \\
\hline AtRbohD-qPCR-R & ATACGGCTCGGCAGTTCACCA & \\
\hline ProAtRbohF-KpnI-F & GGGGTACCTGTAGTCGTCGAGTAGTAACACTT & dual LUC \\
\hline ProAtRbohF-NcoI-R & $\begin{array}{l}\text { CGCCATGGATCCAAAGTCGGAATTCAAAGAGT } \\
\text { TG }\end{array}$ & \\
\hline AtRbohF-qPCR-F & TCTCAGAGCCGACGAAACAACAA & qRT-PCR \\
\hline AtRbohF-qPCR-R & CACCAATGCCAAGACCAACTAATA & \\
\hline AtRbohA-qPCR-F & GAGTCGGAGTGTTTTATTGTGGAG & qRT-PCR \\
\hline AtRbohA-qPCR-R & ATGAACTTGGTGGAGGTCTTATGA & \\
\hline AtRbohB-qPCR-F & ATCGAACGAGGCACTAACCAACAC & qRT-PCR \\
\hline AtRbohB-qPCR-R & CCTCGCATCTCCTTCCTCATACAC & \\
\hline ProAtSEN4-KpnI-F & $\begin{array}{l}\text { GGGGTACCGGTTGCACATATTGATTAGTGATTA } \\
\text { ATATAGTTTTG }\end{array}$ & Dual-LUC \\
\hline ProAtSEN4-SacII-R & $\begin{array}{l}\text { GCGTCCCCGCGGTGTCTTTGTGTGTGTGCGTAC } \\
\text { G }\end{array}$ & \\
\hline $\begin{array}{l}\text { ProAtSEN4-W1- } \\
\text { ChIP-F }\end{array}$ & TGTCCAAAGAAAAGCTCGACGTG & ChIP-PCR \\
\hline $\begin{array}{l}\text { ProAtSEN4-W1- } \\
\text { ChIP-R }\end{array}$ & CCTATTATGGGGTCGTAACTGGAC & \\
\hline $\begin{array}{l}\text { ProAtSEN4-W2- } \\
\text { ChIP-F }\end{array}$ & AATTGACTCCCATCATTAATTAGTGACC & ChIP-PCR/EMSA \\
\hline $\begin{array}{l}\text { ProAtSEN4-W2- } \\
\text { ChIP-R }\end{array}$ & TGGGTTAAAAGATTTGATTGCGTTA & \\
\hline ProAtSEN4-mP2-F & $\begin{array}{l}\text { TTAATTGAATCCCATCATTAATTAGTGACCAAC } \\
\text { CAAAAGAAATTGGACTTTTG }\end{array}$ & EMSA \\
\hline
\end{tabular}




\begin{tabular}{|c|c|c|}
\hline ProAtSEN4-mP2-R & $\begin{array}{l}\text { CAAAAGTCCAATTTCTTTTGGTTGGTCACTAAT } \\
\text { TAATGATGGGATTCAATTAA }\end{array}$ & \\
\hline AtSEN4-qPCR-F & CTCTCGGTGTCTTGTTTCCA & qRT-PCR \\
\hline AtSEN4-qPCR-R & GAAGCCATGAATGGAGCTTT & \\
\hline AtSAG14-KpnI-F & $\begin{array}{l}\text { GGGGTACCGCAAGTATATATACTTGTTGGGTTG } \\
\text { ATTTT }\end{array}$ & Dual-LUC \\
\hline AtSAG14-NcoI-R & $\begin{array}{l}\text { CGCCATGGTGTTATGTGTTGAAGAACTCAGGTC } \\
\text { A }\end{array}$ & \\
\hline AtSAG14-qPCR-F & GGGAGGCATGATGTGGCAGTTGT & qRT-PCR \\
\hline AtSAG14-qPCR-R & GTAGCACCGGCACCAGGAGTAGC & \\
\hline $\begin{array}{l}\text { AtSAG14-W1-ChIP- } \\
\text { F }\end{array}$ & TGTACAATGTCTTTACGCGCT & ChIP-PCR/EMSA \\
\hline $\begin{array}{l}\text { AtSAG14-W1-ChIP- } \\
\mathrm{R}\end{array}$ & TGTGTCGTTGAACAAATCCAAA & \\
\hline ProAtSAG14-mP1-F & $\begin{array}{l}\text { ACTTAGCAGACAAAATTGGACACAAGTGATAA } \\
\text { TATC }\end{array}$ & EMSA \\
\hline ProAtSAG14-mP1-R & $\begin{array}{l}\text { GATATTATCACTTGTGTCCAATTTTGTCTGCTA } \\
\text { AGT }\end{array}$ & \\
\hline AtUBQ10_qPCR_F & GGCCTTGTATAATCCCTGATGAA & qRT-PCR \\
\hline AtUBQ10_qPCR_R & AGAAGTTCGACTTGTC ATTAGAAAGAAA & \\
\hline AtUBC21_qPCR_F & CTTAACTGCGACTCAGGGAAT & qRT-PCR \\
\hline AtUBC21_qPCR_R & GGCGAGGCGTGTATACATTT & \\
\hline ProACT2-ChIP-F & TGCTGGATCTCGATCTTGTTT & ChIP-qPCR \\
\hline ProACT2-ChIP-R & CCATCAAGGTCAAGCCAAAT & \\
\hline ProBnaICS1-KpnI-F & GGGGTACCCATTGTCAGACGCGTGATTT & Dual-LUC \\
\hline ProBnaICS1-NcoI-R & $\begin{array}{l}\text { CGCCATGGGAGATTGCTTAGAGAACGAACAAA } \\
\text { AGAGAG }\end{array}$ & \\
\hline BnaICS1-ChIP-F2 & TCCGCCAATGGTAGTTGAGT & ChIP-qPCR \\
\hline BnaICS1-ChIP-R2 & TCTGTCAAATTGAATCATCGTGGT & \\
\hline BnaICS1-ChIP-F1 & GAGCCACTTTCCTCGCAAA & ChIP-qPCR \\
\hline BnaICS1-ChIP-R1 & ACTTTGCAAATGATGAATCCAATCT & \\
\hline ProBnaPBS3-KpnI-F & $\begin{array}{l}\text { GGGGTACCAGAGACTTACAGTAGAAGATGGAG } \\
\text { AAGG }\end{array}$ & Dual-LUC \\
\hline ProBnaPBS3-NcoI-R & $\begin{array}{l}\text { CGCCATGGTTTTGGTTCTCTCTAATTGATATGA } \\
\text { AGATAGAGAGTGAATG }\end{array}$ & \\
\hline $\begin{array}{l}\text { ProBnaRbohD-KpnI- } \\
\text { F }\end{array}$ & $\begin{array}{l}\text { GGGGTACCGCAAACTTTTAATATACCTTTTCAA } \\
\text { CGGTT }\end{array}$ & Dual-LUC \\
\hline $\begin{array}{l}\text { ProBnaRbohD-NcoI- } \\
\text { R }\end{array}$ & $\begin{array}{l}\text { CGCCATGGTGTTTCAAAGGATGAAAAAAAGAG } \\
\text { ATAATCAC }\end{array}$ & \\
\hline $\begin{array}{l}\text { ProBnaRbohD-ChIP- } \\
\text { F1 }\end{array}$ & TGAAGGCAAACCATAAATGACTCGGT & ChIP-PCR \\
\hline $\begin{array}{l}\text { ProBnaRbohD-ChIP } \\
\text {-R1- }\end{array}$ & GATGGACCGCTCCGGTCC & \\
\hline $\begin{array}{l}\text { ProBnaRbohD-ChIP } \\
\text {-F2 }\end{array}$ & $\begin{array}{l}\text { TGTATATGGCAATTGACTATTTTAGTATATAAA } \\
\text { C }\end{array}$ & ChIP-PCR \\
\hline $\begin{array}{l}\text { ProBnaRbohD-ChIP } \\
\text {-R2 }\end{array}$ & ATCCTCTTTGTTTGACAATTACATAAAATAAAG & \\
\hline $\begin{array}{l}\text { ProBnaRbohD-ChIP } \\
\text {-F3 }\end{array}$ & TGGCTAAGTAGATGTCAATATGTACTAA & ChIP-PCR \\
\hline $\begin{array}{l}\text { ProBnaRbohD-ChIP } \\
\text {-R3 }\end{array}$ & CCTGCACAGTGTGTCAAGC & \\
\hline $\begin{array}{l}\text { ProBnaRbohF-KpnI- } \\
\text { F }\end{array}$ & $\begin{array}{l}\text { GGGGTACCCTATTTGTCTAACCAAAAATTTAAC } \\
\text { ATAAAGCT }\end{array}$ & Dual-LUC \\
\hline
\end{tabular}




\begin{tabular}{|c|c|c|}
\hline $\begin{array}{l}\text { ProBnaRbohF-NcoI- } \\
R\end{array}$ & CGCCATGGGAAGATCCAGCGAACACTAGGGAT & \\
\hline $\begin{array}{l}\text { ProBnaSAG14- } \\
\text { KpnI-F } \\
\text { ProBnaSAG14-NcoI- } \\
\text { R }\end{array}$ & $\begin{array}{l}\text { GGGGTACCTATATATATAAAAATTTAACGATT } \\
\text { AATCCATGTACAATTTACC } \\
\text { CGCCATGGCTTTGTGTTGTGTTGAAAAACTCAG } \\
\text { G }\end{array}$ & Dual-LUC \\
\hline BnaSAG14-ChIP-F2 & GCATTCTGTAAGCCCTCTCCA & ChIP-qPCR \\
\hline BnaSAG14-ChIP-R2 & TGTCCATCCCTACACGTTGA & \\
\hline BnaSAG14-ChIP-F1 & TCAACGTGTAGGGATGGACA & ChIP-qPCR \\
\hline BnaSAG14-ChIP-R1 & AGAGAGGTTTTCAAGGGGTCA & \\
\hline $\begin{array}{l}\text { ProBnaSEN4-NcoI- } \\
\text { R }\end{array}$ & $\begin{array}{l}\text { CGCCATGGTGTTATGTGTGCGTACGTGTGGGTG } \\
\text { TATG }\end{array}$ & Dual-LUC \\
\hline ProBnaSEN4-SalI-F & $\begin{array}{l}\text { CGCGTCGACTTATAGTCTGTCTTAATACCCATT } \\
\text { AATCTAGTTGCATATG }\end{array}$ & \\
\hline BnaUBC21-ChIP-CF & GAACCGTATCCTCTGCAGCCTCCTCAA & ChIP-qPCR \\
\hline $\begin{array}{l}\text { BnaUBC21-ChIP- } \\
\text { CR }\end{array}$ & ATCTTTCGCTTCTTCATTCACAACAA & \\
\hline BnaCPK4_SalI_R & CGCGTCGACGTTTGGTTTGTCATCAGTTTCAGC & $\mathrm{BiFC}$ and $\mathrm{Co}-\mathrm{IP}$ \\
\hline BnaCPK4-SpeI-F & GGACTAGTATGGAGAAACCAAGCTCTAGA & \\
\hline BnaCPK5-EcoRI-F & GGAATTCGGCAATTCATGCCGCCG & GST fusion \\
\hline BnaCPK5-SalI-R & CGCGTCGACGGCGTCTCTCATGCTAATGTTC & $\begin{array}{l}\text { BiFC, Co-IP and GST } \\
\text { fusion }\end{array}$ \\
\hline BnaCPK5_SpeI_F & GGACTAGTATGGGCAATTCATGCCGC & $\mathrm{BiFC}$ and $\mathrm{Co}-\mathrm{IP}$ \\
\hline BnaCPK6-SpeI-F & GGACTAGTATGGGCAATTCATGCCGT & BiFC and Co-IP \\
\hline BnaCPK6-SalI-R & CGCGTCGACAGCATCTCTCATGCTGATG & $\begin{array}{l}\text { BiFC, Co-IP and GST } \\
\text { fusion }\end{array}$ \\
\hline BnaCPK6-BamHI-F & CGGGATCCATGGGCAATTCATGCCGT & GST fusion \\
\hline BnaCPK11-EcoRI-F & GGAATTCATGGAGAAAGCAAATCCTAGAC & GST fusion \\
\hline BnaCPK11-BcuI-F & GGACTAGTGAGAAAGCAAATCCTAGACG & $\mathrm{BiFC}$ and $\mathrm{Co}-\mathrm{IP}$ \\
\hline BnaCPK11-SalI-R & CGCGTCGACATCATCAGACTTTTGAGCG & $\begin{array}{l}\text { BiFC, Co-IP and GST } \\
\text { fusion }\end{array}$ \\
\hline
\end{tabular}

Notes: F and R represent forward (sense) and reverse (anti-sense) primers, respectively. Restriction sites are underlined. 

GAATCAAAACGAGATGTATCTAATGACGTTACCGGCGAGACTCCAACTAGGGTTTCTGCA

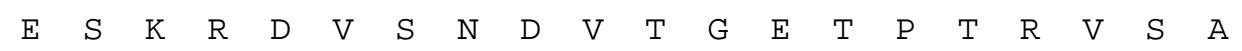
CCTTCTTCTTCTAGCGAGgCTGATCATCCTGgTGAGGATTCCGGTAAAAGTCAGATCAGG $\begin{array}{lllllllllllllllllllll}P & S & S & S & S & E & A & D & H & P & G & E & D & S & G & K & S & Q & I & R\end{array}$ AAAAgAgAgTtAgCtgAAgATgGAggTgAAgAAAATCAAAACTCCAAAAAAGTAGgGAA $\begin{array}{llllllllllllllllllllllllllllllllll}K & R & E & L & A & E & D & G & G & E & E & N & Q & N & S & K & K & V & G & K\end{array}$ ACGAAAAAAAACAAGAGAAGAAACAAAGAGAGCCACGAGTGTCGTTTATGACTAAAAGC $\begin{array}{llllllllllllllllllllllllllllllllll}T & K & K & N & E & E & K & K & Q & R & E & P & R & V & S & F & M & T & K & S\end{array}$ GAAGTTGATCATCTAGAAGATGGTTATAGATGGAGAAAATACGGCCAAAAGgCCGTCAAA

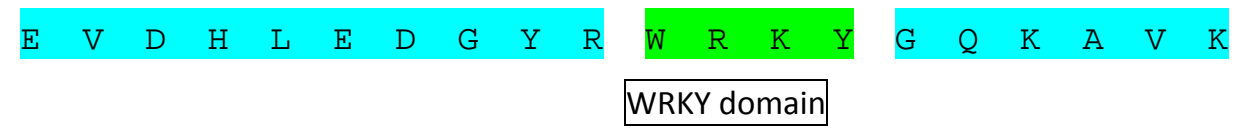
AACAGCCCTTACCCAAGGAGTTACTATAGATGCACGACACAAAAATGTAACGTGAAGAAA $\begin{array}{llllllllllllllllllll}N & S & P & Y & P & R & S & Y & Y & R & C & T & T & Q & K & C & N & V & K & K\end{array}$ CGAGTGGAGAGgTCGTTCCAAGATCCAACGgTTGTGATTACAACGTACGAGGGTCAACAC $\begin{array}{llllllllllllllllllll}R & V & E & R & S & F & Q & D & P & T & V & V & I & T & T & Y & E & G & Q & H\end{array}$ AACCATCCCATTCCGACTAATCTTCGGGGAAATTCCGCGGCGGCTGCTATGTATTCTGAC $\begin{array}{llllllllllllllllllll}N & H & P & I & P & T & N & L & R & G & N & S & A & A & A & A & M & Y & S & D\end{array}$ TTCATGACTCCGAGAAGCTTTACACATGATATGTTTCGGACGGCTGCTTATACTAGCGGT $\begin{array}{lllllllllllllllllllll}\mathrm{F} & \mathrm{M} & \mathrm{T} & \mathrm{P} & \mathrm{R} & \mathrm{S} & \mathrm{F} & \mathrm{T} & \mathrm{H} & \mathrm{D} & \mathrm{M} & \mathrm{F} & \mathrm{R} & \mathrm{T} & \mathrm{A} & \mathrm{A} & \mathrm{Y} & \mathrm{T} & \mathrm{S} & \mathrm{G}\end{array}$ GGCTCTGTGGAGGGGGCTTTGGATTATGGATACGGACAGAGTGGTTATGGTAGTGTGAAT

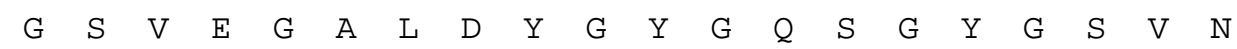
GCAAACCCTAATTCTTCTCATCAAAACTATCACCAAGGAGGCGAATATGAGCTTTTGAAG

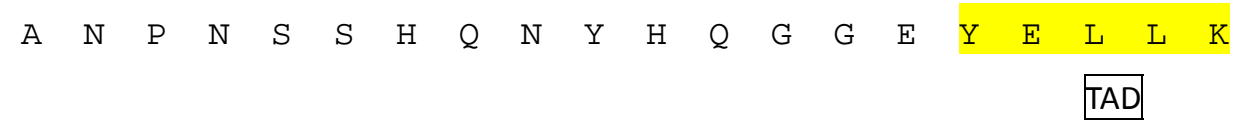
GAAATTCTTCCTTCCATTTTCTTCAAGCAAGAGCATTGA

Fig. S1 The coding region and translated sequence of BnaWSR1.

The conserved peptide stretch of WRKY is highlighted in green, while the WRKY domain in blue. Potential zinc ligands are highlighted in pink. The 9-AA transcriptional activation domain (TAD) at the C-terminus is highlighted in yellow. The two Thr (T) residues, which are putative phosphorylation sites of CPK are 
highlighted in red.
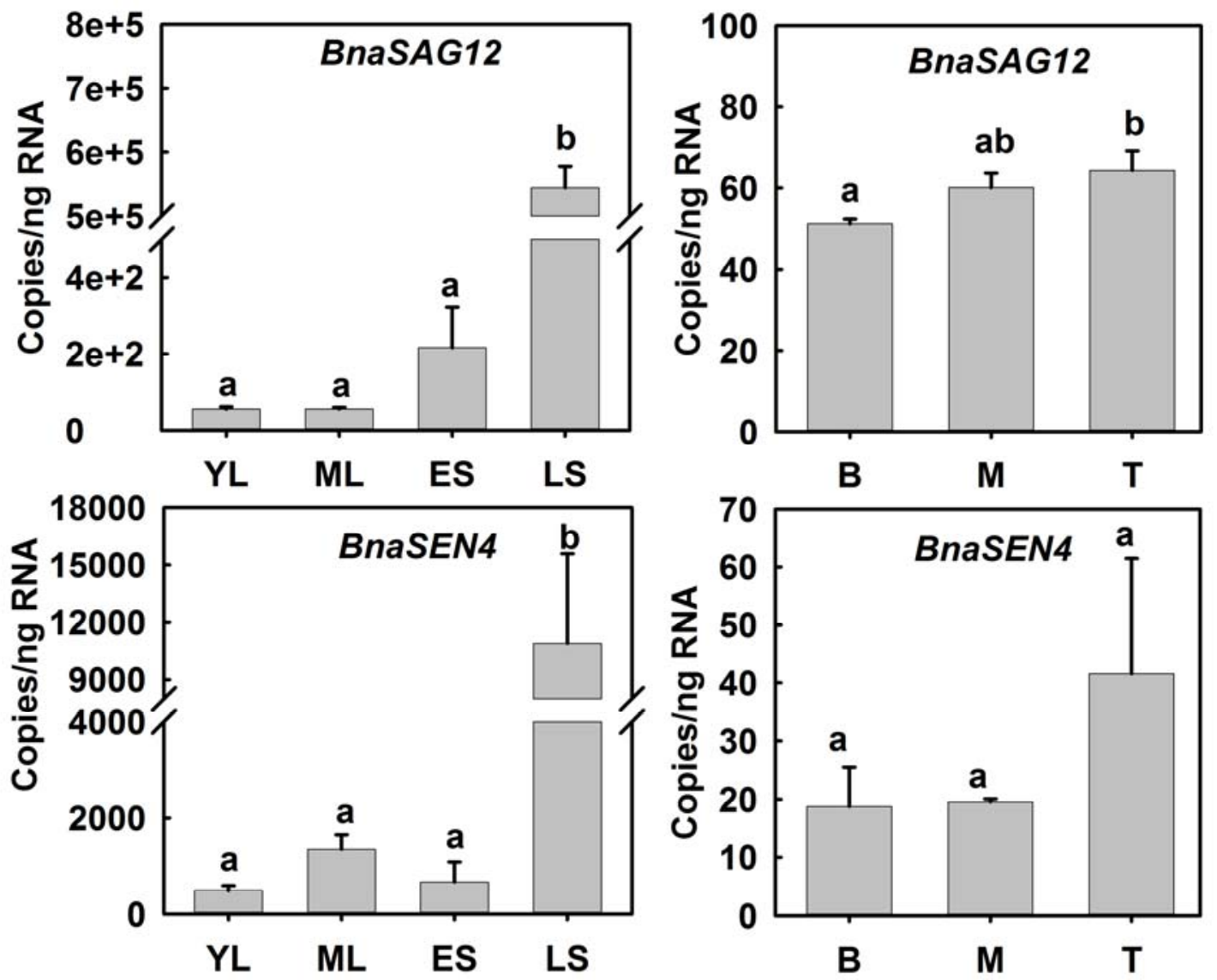

Fig. S2 Analysis of expression of two senescence marker genes during leaf development.

Expression of BnaSAG12 and BnaSEN4 in the fourth true leaves of rapeseed at four different developmental stages. YL, young leaves of 21-day-old seedlings; ML, fully expanded mature leaves of 28-day-old seedlings; ES, leaves of 35-day-old seedlings at early senescent stage; LS, leaves of 44-day-old seedlings at late senescent stage, with $>50 \%$ leaf area yellowing. The right panel shows the expression levels of BnaSAG12 and BnaSEN4 in different part of an early senescing leaf. B, base; M, middle; T, tip. Data represent means of three biological replicates \pm standard errors $(\mathrm{SE})$. Different letters indicate significant differences $(P<0.05)$. 


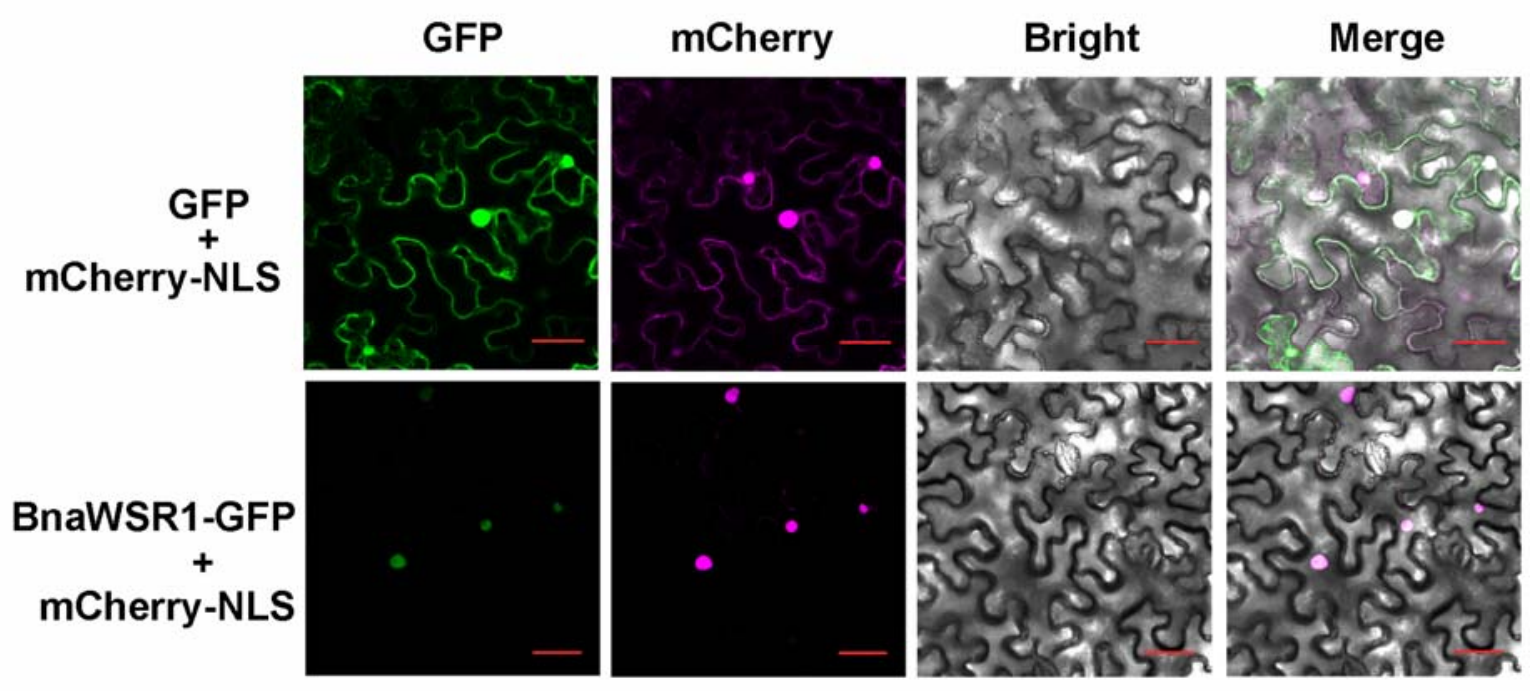

Fig. S3 Subcellular localization of BnaWSR1.

Subcellular localization of GFP and BnaWSR1-GFP in epidermal cell of tobacco leaves. A plasmid containing NLS-mCherry was co-transformed as a nuclear marker. GFP, mCherry, bright and merge represent the GFP, mCherry fluorescence, bright and merged fields, respectively. Bars, $50 \mu \mathrm{m}$. 

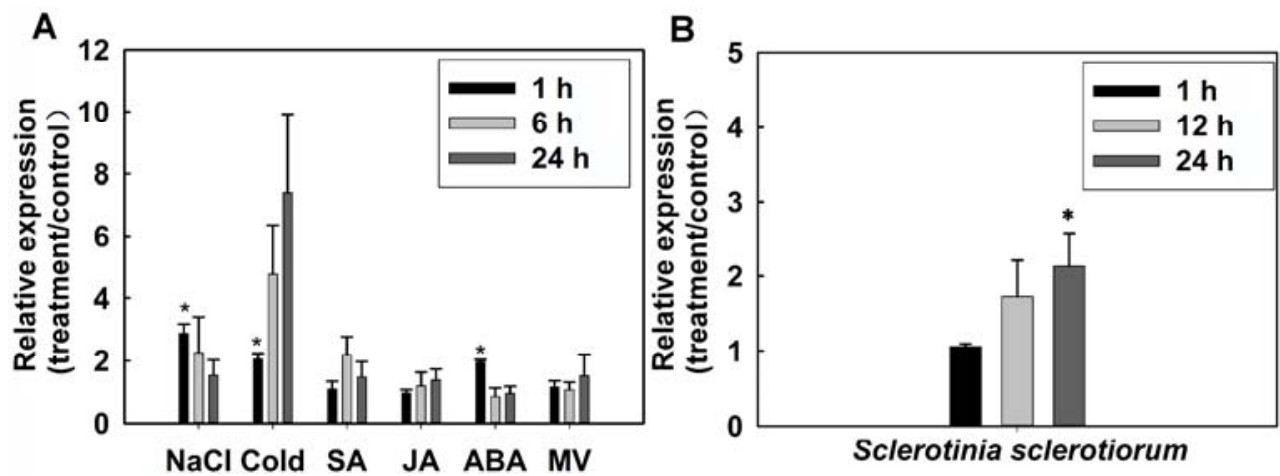

Fig. S4 Expression of BnaWSR1 in response to various treatments.

Seedlings of 7-day-old were treated with $2 \mathrm{mM}$ salicylic acid (SA), $100 \mu \mathrm{M}$ jasmonic acid (JA), $50 \mu \mathrm{M}$ absicic acid (ABA), $10 \mu \mathrm{M}$ methyl viologen (MV), $200 \mathrm{mM} \mathrm{NaCl}$, cold $\left(4^{\circ} \mathrm{C}\right)$ for $1 \mathrm{~h}, 6 \mathrm{~h}$ and $24 \mathrm{~h}$ while 18 d-old seedlings were inoculated with Sclerotinia scleoriotum for $1 \mathrm{~h}, 12 \mathrm{~h}$ and $24 \mathrm{~h}$. Data are the mean of three biological replicates \pm SE. Asterisks denote significant differences (compared with 1 ) by Student $t$-test analysis $(* P<0.05)$. 
AtWRKY42

AtWRKY6

AtWRKY28

BnaWSR1

AtWRKY8

AtWRKY71

AtWRKY45

AtWRKY75

AtWRKY48

AtWRKY57

AtWRKY26

AtWRKY22

AtWRKY27

AtWRKY65

AtWRKY46

AtWRKY38

consensus

301

AtWRKY42

AtWRKY6

AtWRKY28

BnaWSR1

AtWRKY8

AtWRKY71

AtWRKY45

AtWRKY75

AtWRKY48

AtWRKY57

AtWRKY26

AtWRKY22

AtWRKY27

AtWRKY65

AtWRKY46

AtWRKY38

consensus
256

281

136

130

148

100

39

41

185

120

198

103

133

48

76

82
NGSENASSKVIEQAAAEATMRKARVSVRARSEAPMLSDGCQWRKYGQKMAKGNPCPRAYY - - - - STTPTTFDQTAEATMRKARVSVRARSEAPMISDGCQWRKYGQKMAKGNPCPRAYY EEDQISKKVGKTKKTEVKKQREPRVSFMTKSEVDHLEDGYRWRKYGQKAVKNSPYPRSYY EENQNSKKVGKTKKNEEKKQREPRVSFMTKSEVDHLEDGYRWRKYGQKAVKNSPYPRSYY EDDQRSQKVVKTKKKEEKK - KEPRVSFMTKTEVDHLEDGYRWRKYGQKAVKNSPYPRSYY VGESSKQLTKQGKKKGEKKEREVRVAFMTKSEIDHLEDGYRWRKYGQKAVKNSPYPRSYY - . - . - - - KPRSKKKKKEREARYAFQTRSQVDILDDGYRWRKYGQKAVKNNPFPRSYY - - - - - - - - KSVESSKKKGKKQRYAFQTRSQVDILDDGYRWRKYGQKAVKNNKFPRSYY QEQKGTKPQLKAKKKNQKKAREARFAFLTKSDIDNLDDGYRWRKYGQKAVKNSPYPRSYY - - - - - - - -VKEKKKAQKRIRQPRFAFMTKSDVDNLEDGYRWRKYGQKAVKNSPFPRSYY GKDIGEDETEAKRWKREENVKEPRVVVQTTSDIDILDDGYRWRKYGQKVVKGNPNPRSYY - - - - - - - - SNTSRSKRRKIQHKKVCHVAAEALNSDVWAWRKYGQKPIKGSPYPRGYY - - - LLQQQSQPPLRSRKRKNQQKRTICHVTQENLSSDLWAWRKYGQKPIKGSPYPRNYY - . - . - . - - SVEKRVVNVPMKEMEGSRHKGDTTPPSDSWAWRKYGQKPIKGSPYPRGYY - - - - - - VFKKRKVSEKNTEKVKVFVATEQENGSIDDGHCWRKYGQKEIHGSKNPRAYY - - - - - - QGSPSKKRKIDSTNSSENWRDDSPDPIYYDGYLWRKYGQKSIKKSNHQRSYY k kkk kk $r$ rv $f$ trsevdmleDgyrWRKYGQKavknspypRsYY * *

316

336

196

190

207

160

89

91

245

171

258

152

189

98

128

134

361

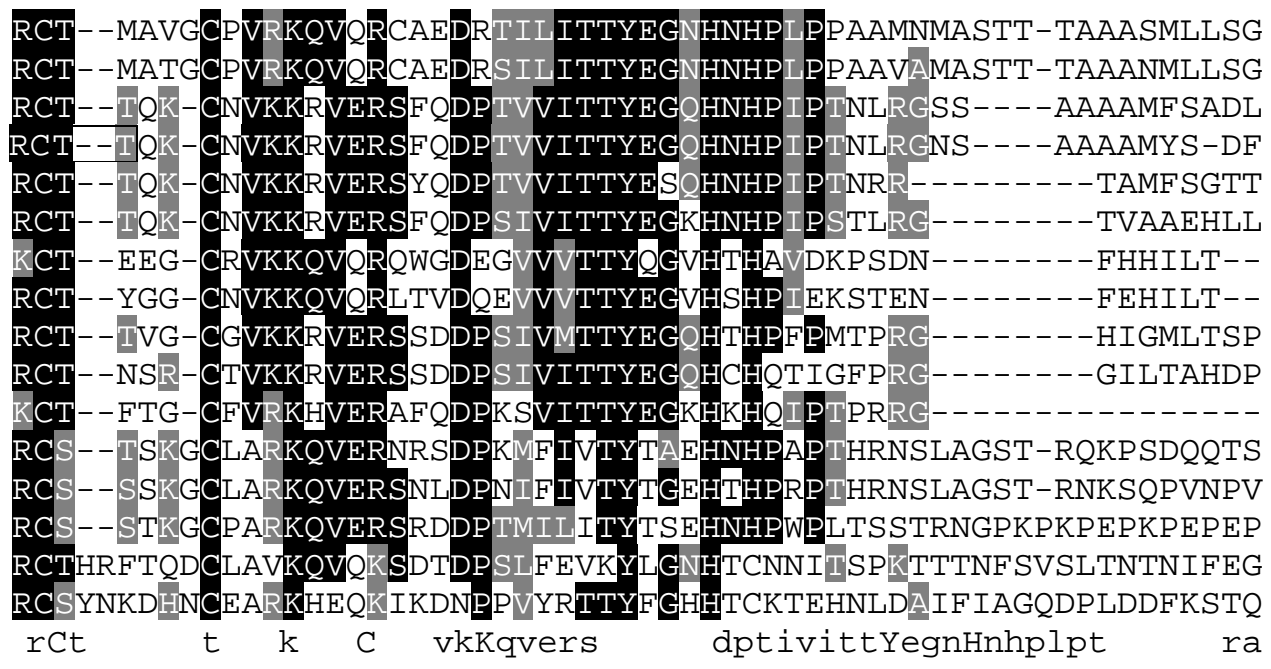

Fig. S5 Alignment of DNA binding domains of BnaWSR1 and representative AtWRKYs. Identical amino acid residues are shaded in black, and similar amino acid residues are shaded in gray. The conserved Threonine (T) residues in BnaWSR1 and AtWRKY28, and -8 are indicated by asterisks above the alignment. 


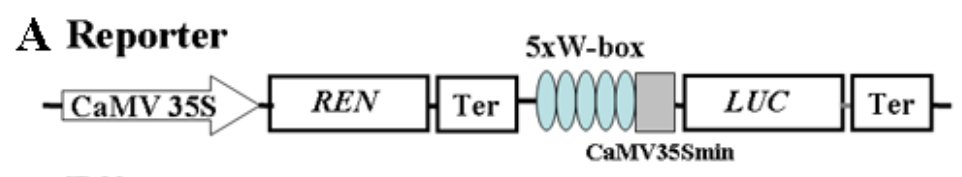

\section{Effector}

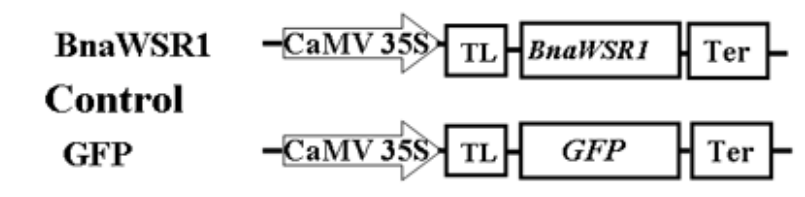

B

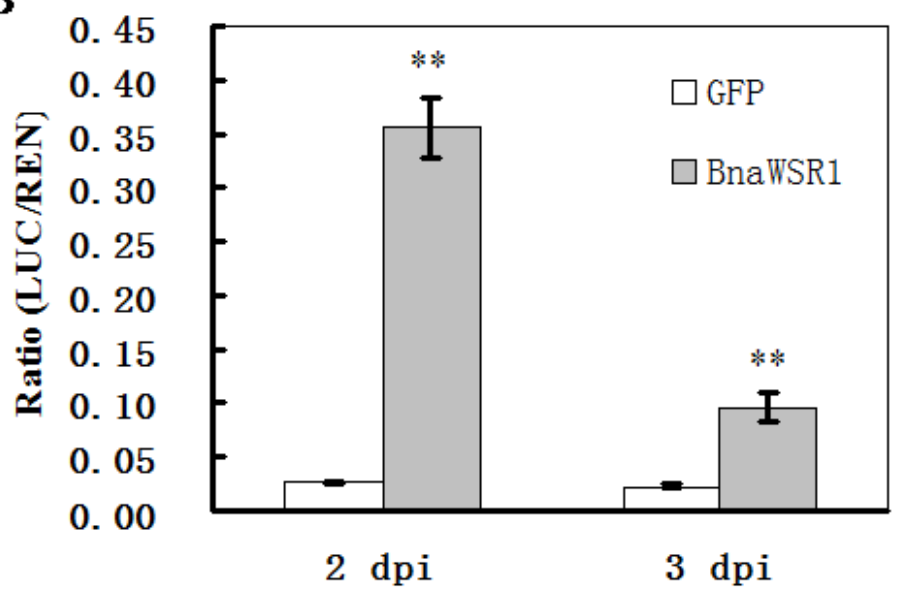

Fig. S6 Transcriptional activity assay of BnaWSR1.

(A) The schemes of expression cassettes of various plasmids. 5x W-box indicates five tandem repeats of canonical W-box sequences, which were fused with minimal sequence of CaMV35S promoter. TL, translational leader. Ter, terminator sequence. LUC, firefly luciferase; REN, Renilla luciferase. (B) The transcriptional ability of BnaWSR1 to five tandem repeats of canonical W-box sequences. The LUC/REN ratios of BnaWSR1 and GFP were determined from three independent replicates at two time points. Dpi, days post-infiltration. Each value represents the mean \pm SE from three independent biological replicates. The asterisk indicates a significant differences in LUC/REN ratio $(* *, P<0.01)$ compared to the GFP control effector. 


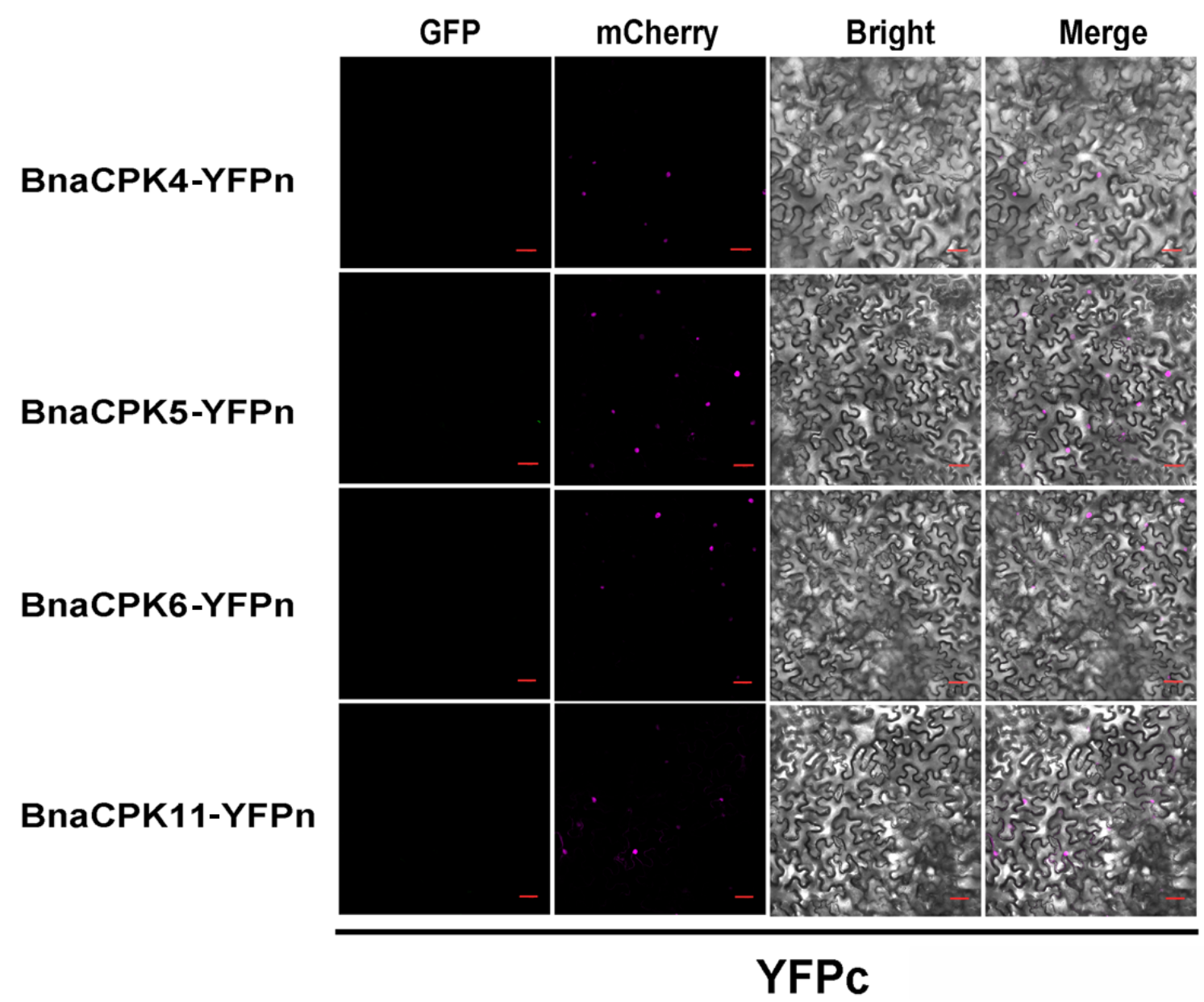

Fig. S7 Negative controls of the BiFC assay.

Agroinfiltration was performed to coexpress BnaCPK4/5/6/11-YFPn and YFPC in leaves of tobacco. $3 \mathrm{~d}$ later, samples were observed on a confocal microscope. The fields from left to right are YFP, NLS-mCherry fluorescence, bright and a merge of three fields, respectively. Scale bars, $50 \mu \mathrm{m}$. 


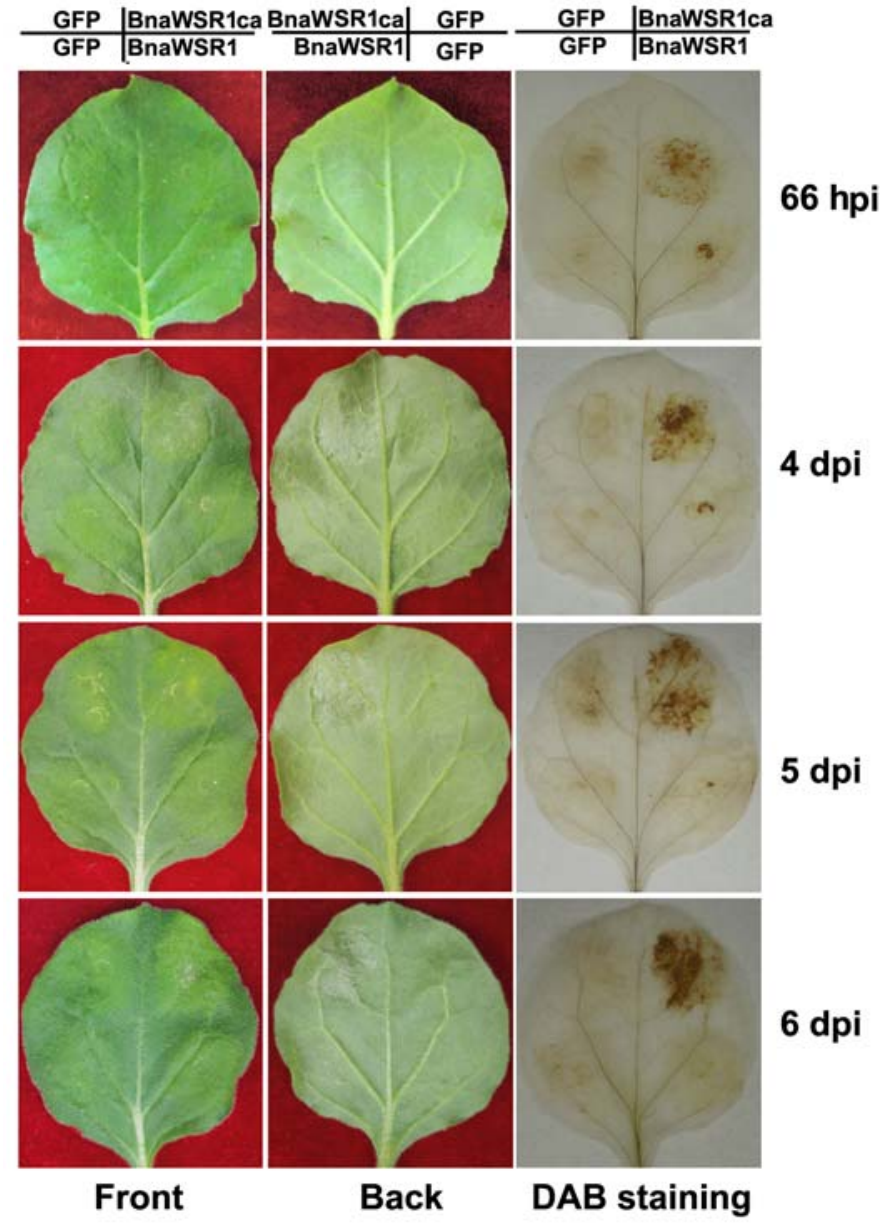

Fig. S8 Examination of BnaWSR1 function in transient expression assay in tobacco. Cell death symptom and ROS production were observed in leaf epidermal cells of tobacco expressing native and phosphomimic BnaWSR1 from 66 hpi (hours post-infiltration) to 6 dpi (days post-infiltration). DAB staining was done to show $\mathrm{H}_{2} \mathrm{O}_{2}$ accumulation. GFP was expressed as a control. 


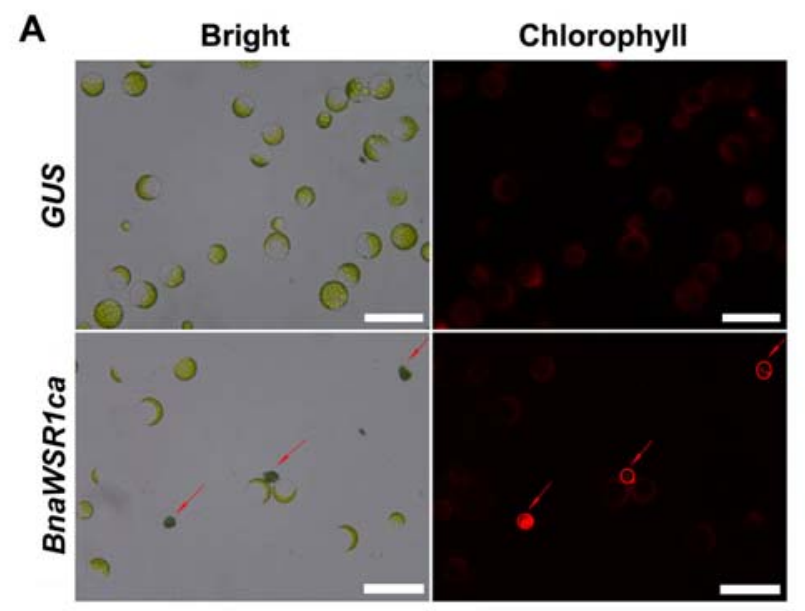

B

C

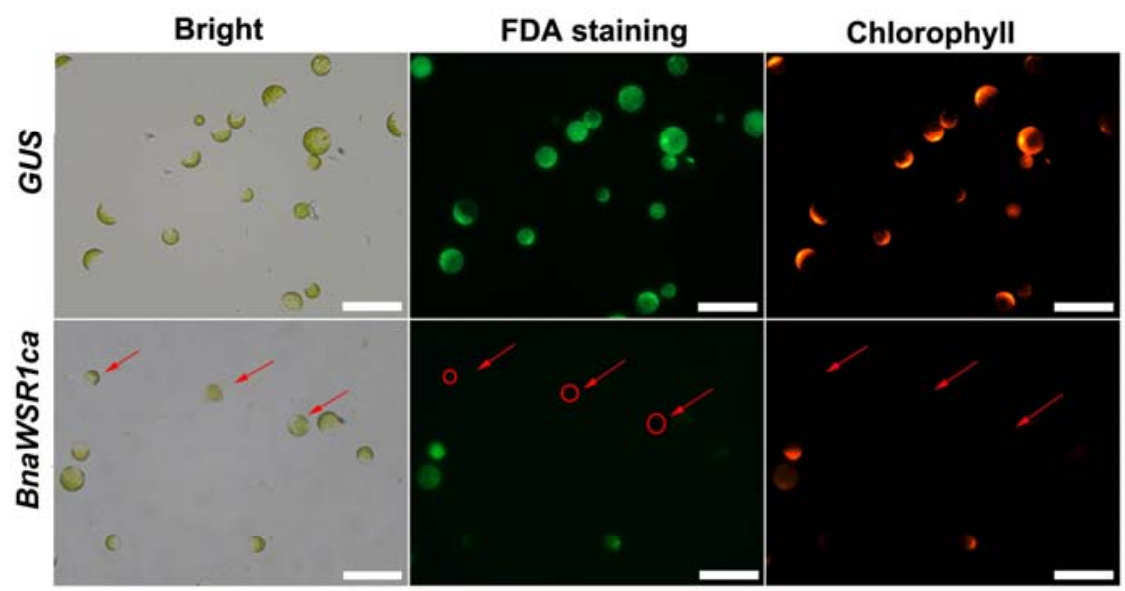

D

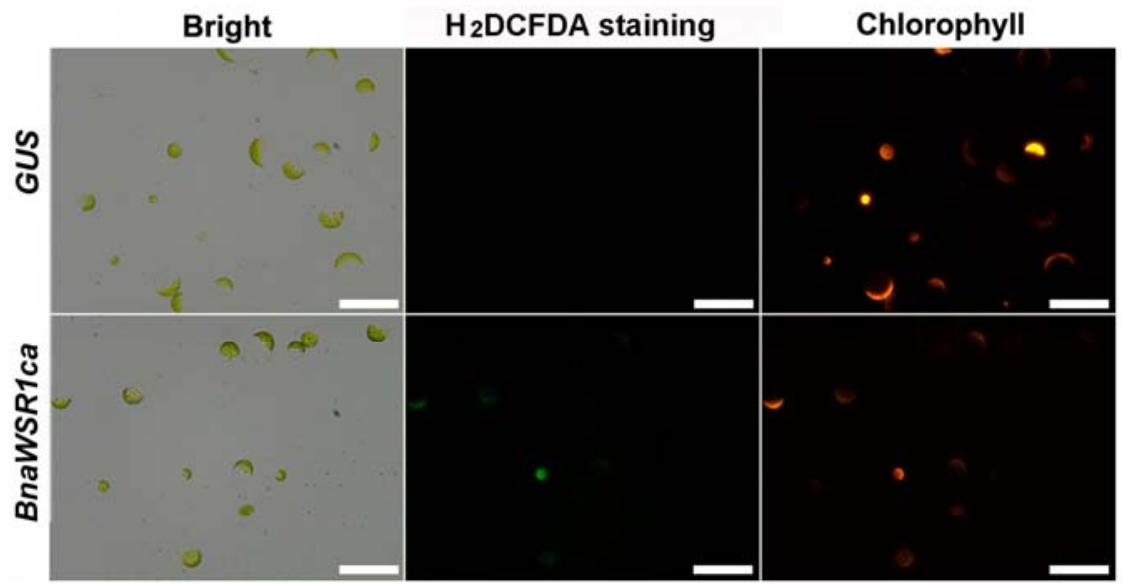

E

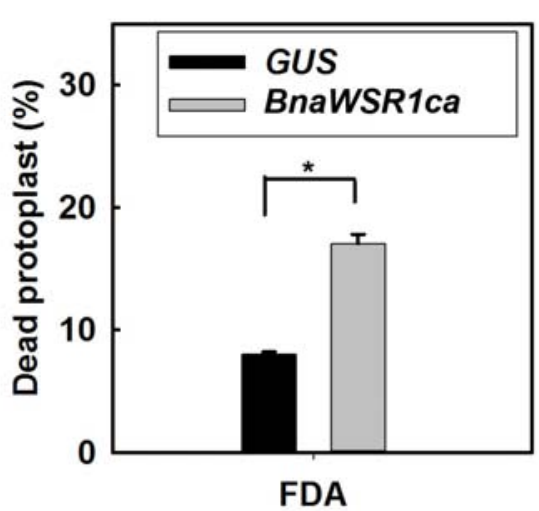

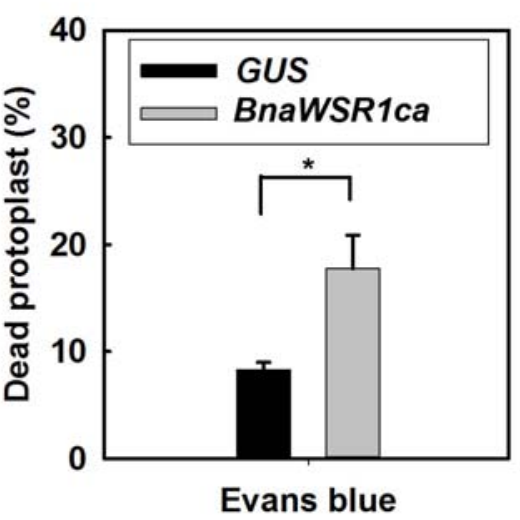

Chlorophyl

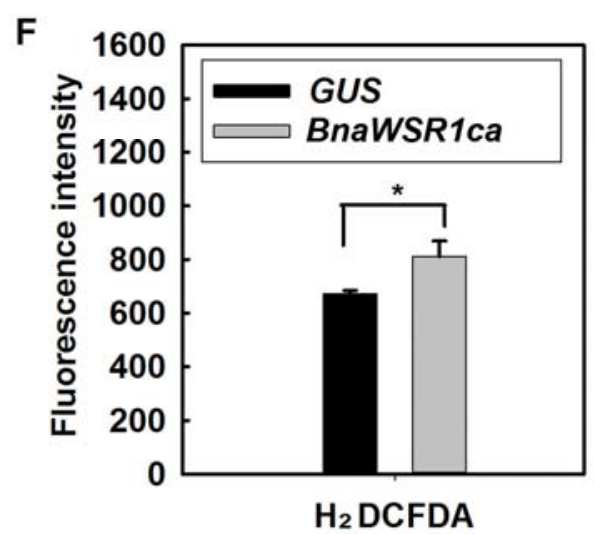


Fig. S9. Expression of BnaWSR1ca in rapeseed protoplasts resulted in ROS accumulation and cell death. (A-B) Evans blue staining and comparison of dead protoplast percentages. Dead protoplasts were stained with Evans blue dye and indicated by arrows. Bars, $100 \mu \mathrm{m}$. Data are mean of three replicates $\pm \mathrm{SE}$ (n $=20)$. Asterisks indicate the significant difference by Student's $t$-test $\left({ }^{*} P<0.05\right)$. (C) FDA staining. The arrows indicate dead protoplasts. (D) $\mathrm{H}_{2} \mathrm{O}_{2}$ accumulation indicated by $\mathrm{H}_{2}$ DCFDA staining. The green fluorescence in the middle panel indicates $\mathrm{H}_{2} \mathrm{O}_{2}$ accumulation. Bars, $100 \mu \mathrm{m}$. Autofluorescence of chloroplasts was shown in the right panel in A, C and D. (E-F) Percentages of dead protoplasts as detected by FDA (E) and $\mathrm{H}_{2}$ DCFDA (F) stainings, respectively. Data are mean of three replicates $\pm \mathrm{SE}(\mathrm{n}=20)$. Asterisks indicate the significant difference by Student's $t$-test $\left({ }^{*} P<0.05\right)$. Scale bars, $100 \mu \mathrm{m}$. 


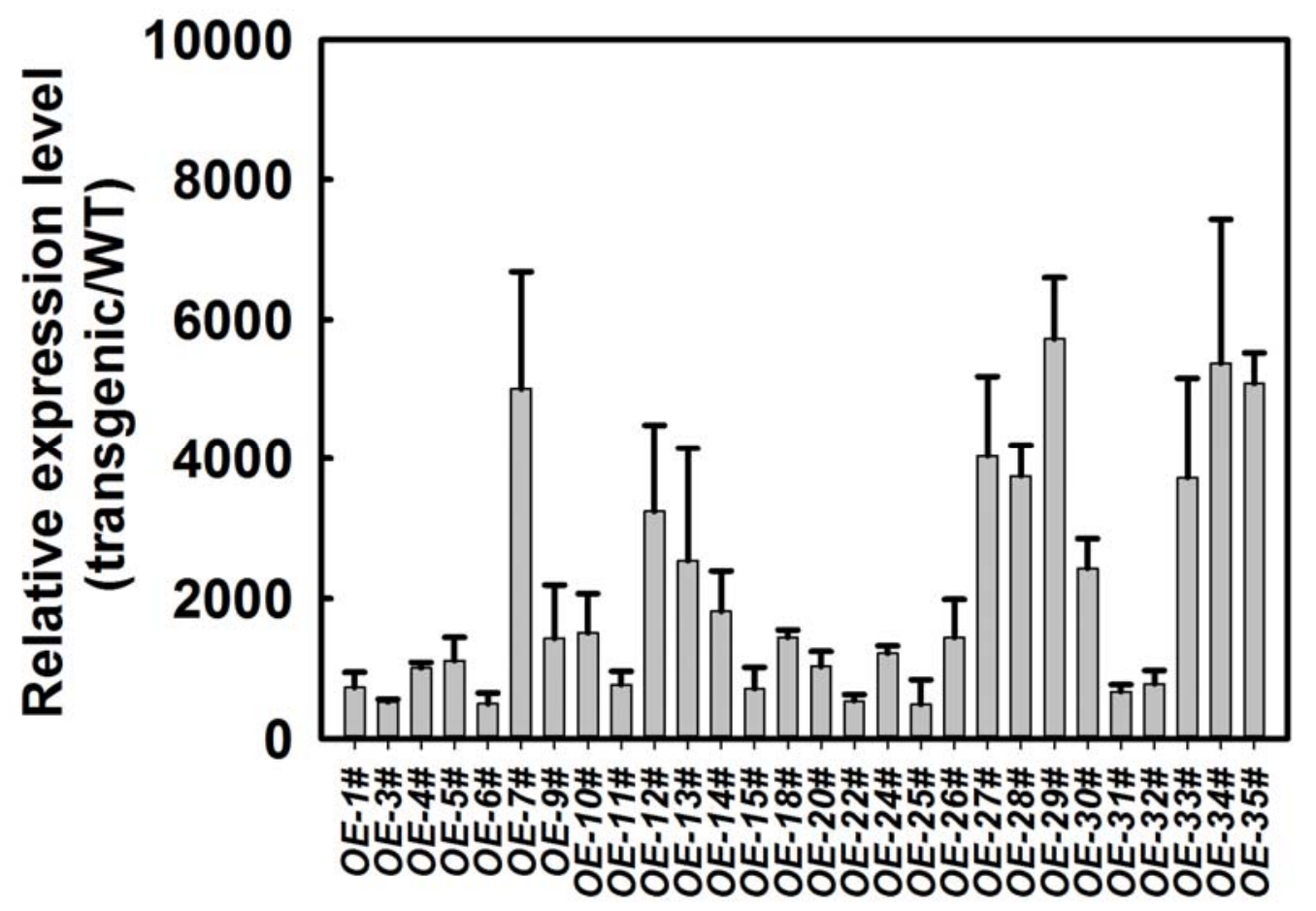

Fig. S10 qRT-PCR analysis of BnaWSR1 level in constitutive overexpression plants.

RNA was extracted from 7-day-old plants grown on 1/2 x MS media, and transcript level of BnaWSR1 was assayed through qRT-PCR and was compared to that in Col-0 seedlings. AtUBC21 and AtUBQ10 were used as internal controls. Values are means $\pm \mathrm{SD}$ of two independent replicates. 


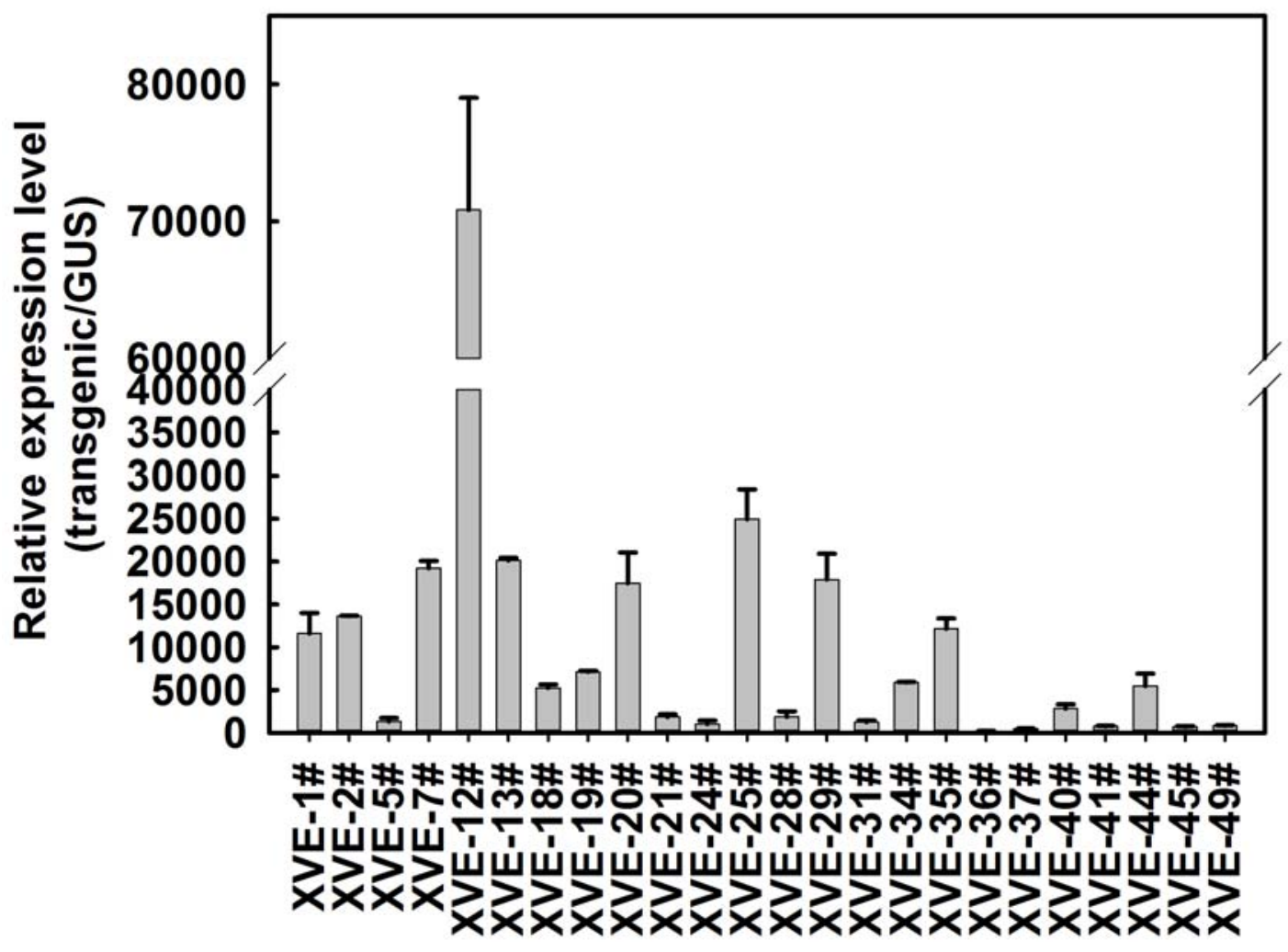

Fig. S11 Expression analysis of BnaWSR1 in XVE-BnaWSR1ca transgenic lines through qRT-PCR.

RNA was extracted from 7-day-old plants grown on $1 / 2 \times$ MS medium that had been induced by $5 \mu \mathrm{M} \beta$ estradiol for $2 \mathrm{~d}$, and transcript level of BnaWSR1 was assayed through qRT-PCR and was compared to that in the similarly induced transgenic pER8-GUS seedlings. AtUBC21 and AtUBQ10 were used as internal controls to normalize the data. Values are means $\pm \mathrm{SD}$ of two independent replicates. 


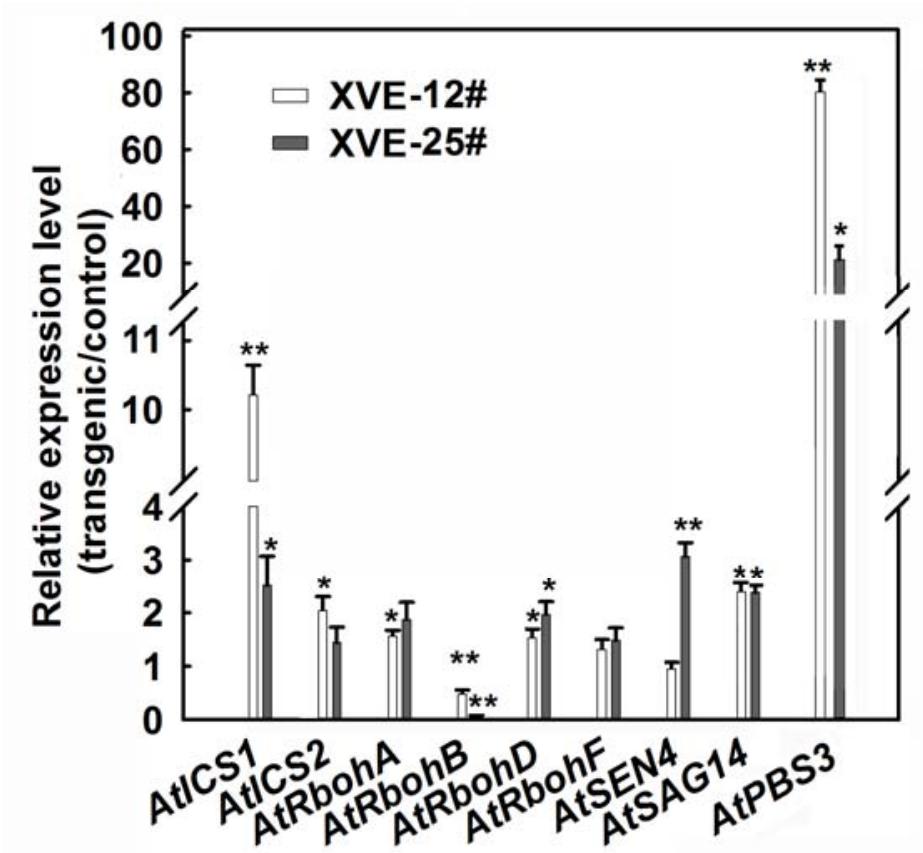

Figure S12. Expression analysis of SA and ROS production associated marker genes and senescencerelated genes. Two independent homozygous T3 lines (12\# and 25\#) of inducible BnaWSR1ca overexpression were used for qRT-PCR analysis after induced with $5 \mu \mathrm{M}$ estradiol for $2 \mathrm{~d}$. Values were means $\pm \mathrm{SE}$ of three independent biological replicates with 20 seedlings for each genotype per replicate. Asterisks indicate significant differences in fold change $(*, P<0.05 ; * *, P<0.01)$ relative to pER8-GUS transgenic line by Student's t test. The expression level of each gene in pER8-GUS control line was set as 1 . 

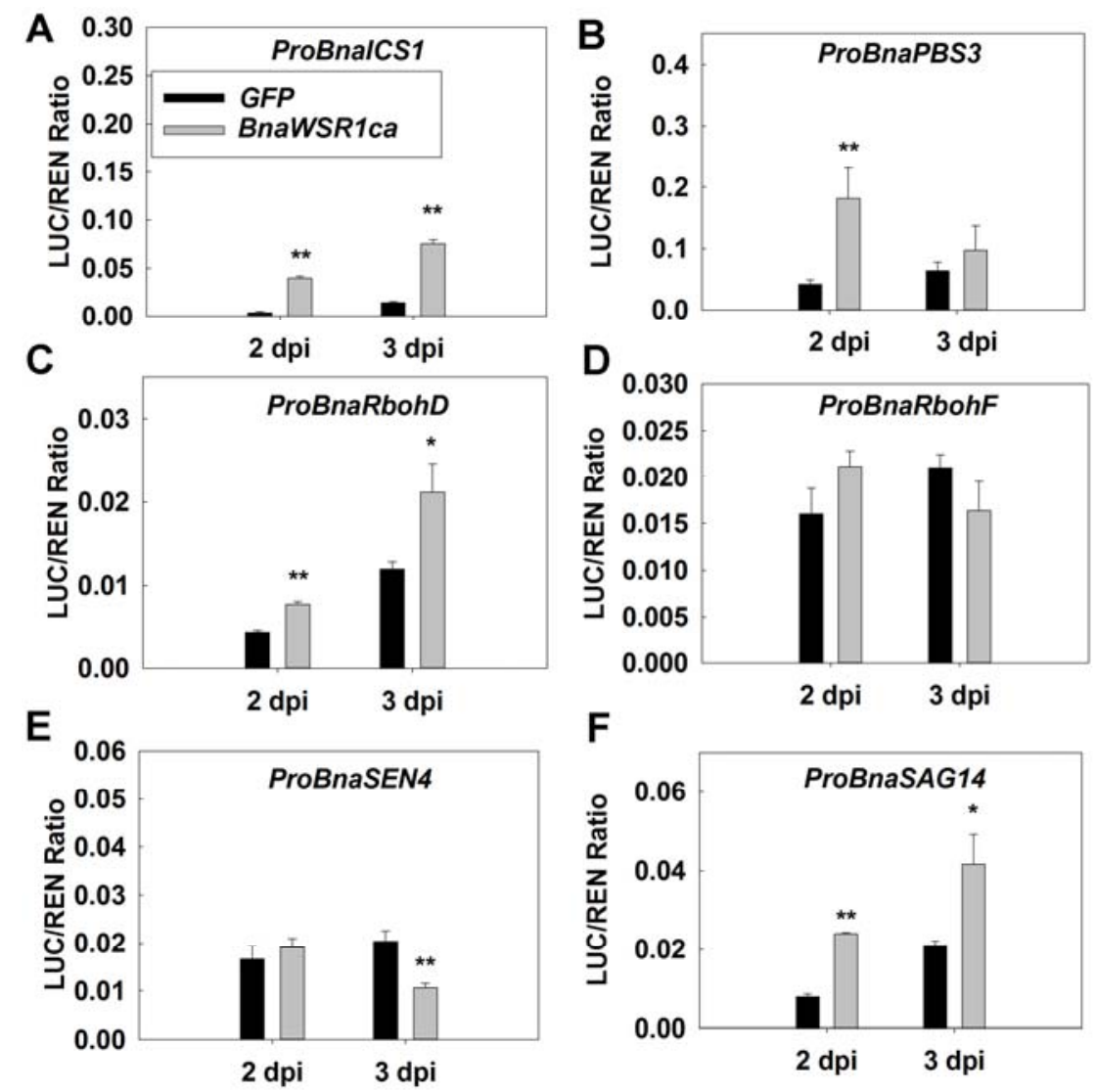

Fig. S13 Regulatory effect of BnaWSR1ca on the promoters of rapeseed genes.

The transcriptional ability of BnaWSR1ca to activate the expression of putative target genes in rapeseed is reflected by the LUC/REN ratios. Different combinations of effectors and reporters were co-expressed in tobacco leaves for 2 and 3 dpi (days post-infiltration) before sampled. Values were means \pm SE of three independent biological replicates. Asterisks indicate significant differences $(*, P<0.05 ; * *, P<0.01)$ compared to the GFP control effector. 


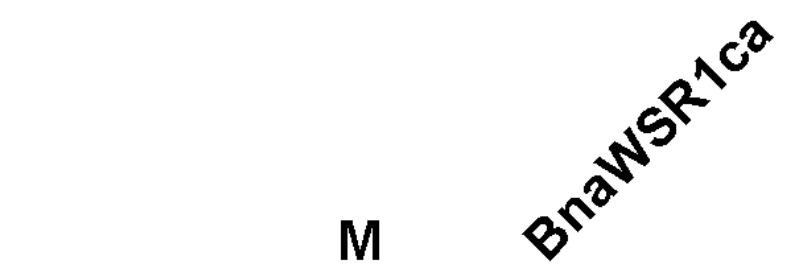

$70 \mathrm{kDa}-$

$60 \mathrm{kDa}-$

$50 \mathrm{kDa}-$

$40 \mathrm{kDa}-$

Fig. S14 Inspection of purified GST-BnaWRS1 protein via SDS-PAGE.

Purified GST-BnaWSR1was separated in 10\% SDS-PAGE gel followed with staining by Coomassie Briliant Blue (CBB). An unstained protein ladder $(\mathrm{M})$ is loaded in Lane 1 and the purified fusion protein of GST-

BnaWSR1 is loaded in lane 2. The molecular weights of bands of the protein ladder are shown at the left. The molecular weight of GST-BnaWSR1ca fusion protein is $60.986 \mathrm{kDa}$. 


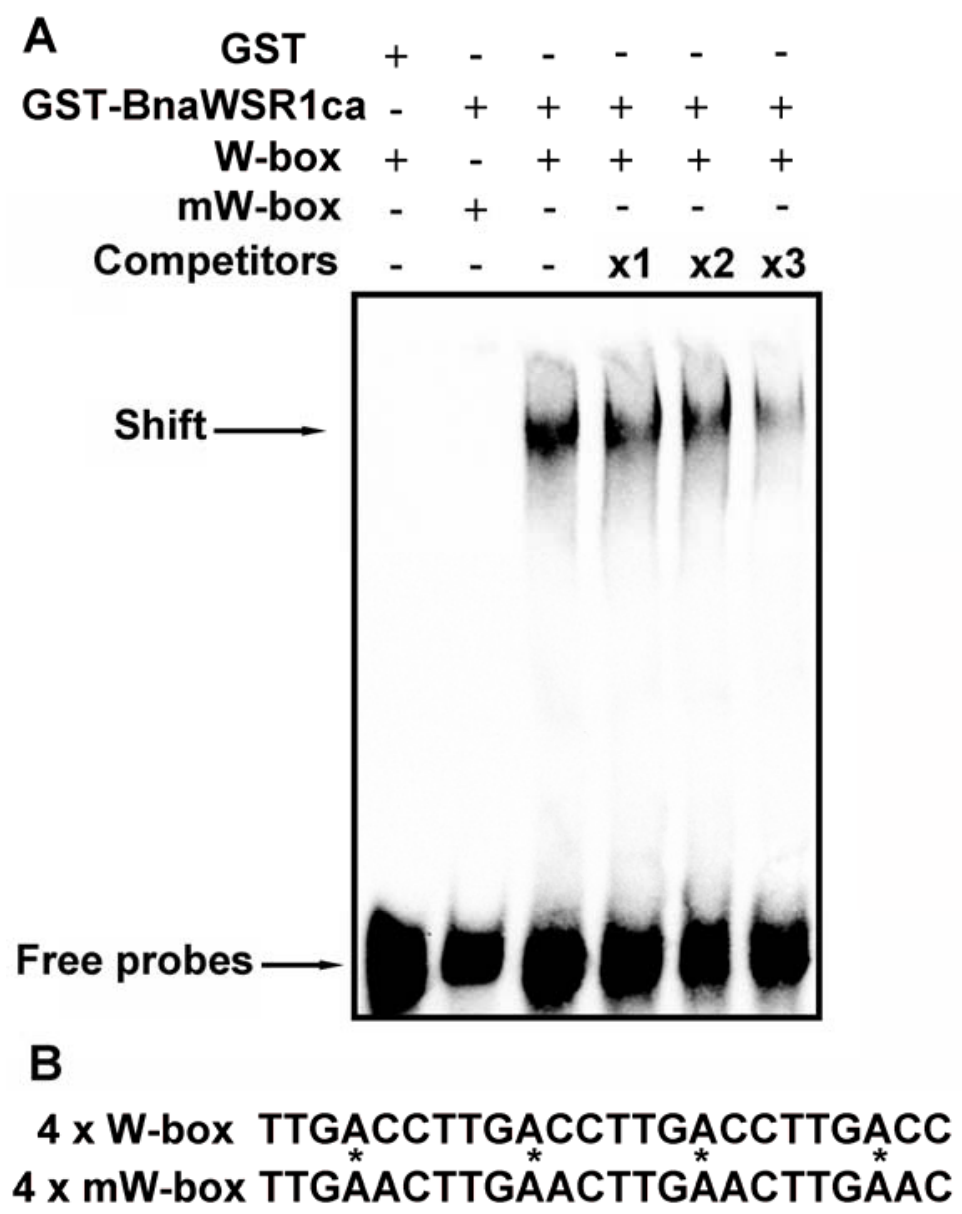

Fig. S15 EMSA assay of binding of phosphomimic BnaWSR1 to the W-box.

(A) In vitro binding of BnaWSR1 to W-box. Competitive probes of unlabeled probes were added in molar excess as shown above the lanes. Mutated W-box (mW-box) was run as a control. The bands at the upper and lower part of the membrane indicate protein-DNA shift and free probes, respectively. '+'and '-' indicate the presence and absence of one component in binding reactions, respectively. (B) The sequences of tandem repeated W-box and mutated W-box. Asterisks indicate mutated sites. 
>AtICS1 (AT1G74710) promoter: contains 4 canonical W-boxes, $2.1 \mathrm{~kb}$

Actcagactccagaagacgactttatttttcccaaattgttttatactcaaaggaataaattatccacgctttgtcacacaaaaaaaataaaaattatatagatatata gatatatacatgcaggaatttaaataaaattattaatcttacgtaattaagaacttttttattttttttaaaaatcgtaattaagaacttgaatggtaaaatactagaagaa

ProAtICS1-ChIP-F3

>>>>>>>>>>>>>>>>>

aattgttagcgtcagtgtgaaagcaaattatactgtaggttaacttttctatagtgagcaaaatacattaggttatctaaattcaactaacgtcctatttctagttatttg

W4

ProAtICS1-ChIP-R3

W4 agacttaagtctagaaggatgacggaaatgagaataagctaaaacgtttctacgaaattaagaaattcgtagcatccacaacacacatattcaaagtcttgaataa

caataaaattatgttcagctgagagtctgagaccgcgtccaacattttaaaacacttttaaaagtcatactataatatctgaggaccgtgtctgtttcagtttattcggtt ProAtICS1-ChIP-F2

>>>>>>>>>>>>>>>

AtICS1-P3 (W3)

ttacaacagcaatgatgcttgttgaattatggtttcattctattggattatctgcaagacttcttaattttaatatatagctcgtgatgataaaataacaatttgacttctaaa

W2

ProAtICS1-ChIP-R2

$<<<<<<<<<<<<<<<<<$

gtctagtcctttatagtcttaacaatattcattttgaccaggttgagtaa]atcagacaaaaatctaaagacaaagaataatcgtttcctcaaatatgtacttggtgagc cgtcttaatcaccatatgtacatgagaataaatgtgtaatttggtggttatttcatagaaattttggggaaattgttgcacctctccactctttgccagtctcgtgtgatc

ttaattgactcaaaatgaaagaaaaaaaacatgaaattaaagcaaatattcctatttgaattatgttcctttaagatttttaacaatattttttaaatgataaaaactggt ctcaaagagcctaagtgggtttcctagcaaaaaaagatactcaaagtaatttactcctcaaaataatgcaatgatctaatgaagatctgttcaaatagtattgattttc caaatttaattatactcaaagagttttaaaatctgttcttgttattctatgctttgttttacatgtaaagtacttaaattcacttgatatttgttattactataattttagaaatgtg tcaaaatttaaaggagcatgcgtgtaatgccatatgccttatgtacgagaacttgtaatgcgtttgcaatttgtagtgacatgtaatgttttttacgatagtttaaagtgc aaaccgcttccgtatcaaacgagaagagtcgtctagcatacaacacccacacgagaggaaccgcttagaaagagaatccacttgaaattgctgtccatgcattg ctcaaattcattgatacttggtctatgcaattttacactacctcatttctgactatttcaatttgtcaagatattataacatgcatttataactttttgtcgacgataaatcaa ccaaacgaatccggtctgtatgtttgataacatgcattcatacaaaacgattatctgttttttagtctatttaatttggtttctactttttacttttgtgaaactaataataata gatcaaacaattaactgatataatttattaatttaattcttagaccaagtaaatgattcatgaaatattaaatgcacgactaactttagaaaaatgtttctttgtatacataa agatagaatagaagaaaagtaggattagaagaaaaaaacgaaaatttcagagtagtttactaaagaaattctgagaaattttgtgtgaaaatgaaatgaaaatctt caattttagtgggeccetgctacatcagtcccctatttatatctcttctcaactctaaacccaaaccaaaaaaaaacagaaagagcaatctctctatactacaaacac caaagtttaaaattaaacaaaatcttcaagaaacactttacgaatttctgcaATG

AtICS1-P3 gataaaataacaatttgacttctaaagtctagt

AtICS1-mP3 gataaaataacaatttggattctaaagtctagt

>AtPBS3 (At5g13320) promoter: contains 5 canonical W-boxes, $2.6 \mathrm{~kb}$

Cagggcaactagaagcaatccataaatattattacgattacaaaatttgcttaagaggattaatcaaaatatttttgtgaaaaatatttcaaatattaactttcattacaa ataaaggatttttacccccaaaaaaaaataaagtacttttataattaaaccaaaatattataattacctaattataaatgaatattttatgattatcttacataaaagatc W5

Gatgtttacaaaacaaatcattttcaaaagaaaaataagtacattaaataataagatccctttgatttatggtttatttagaaagtcaaaacgcgaaaatgttcgaaa atattataaagccatttattcagtagatgtaatgtcttatacggccegagaagcggatgcgttccgcgtagcaattgctcgggettgcatggacgctacttaaatcg acacgattcatactatattgtctgtcaaatcgatagctcgtgggacgtatcagattcattaagaggcgatggatggettgtcctttctcgctagaatattgccetcctc gggttaaagagctaccacagataaatgtcaccctacacacatagttcgaatatctcttttgggcaataaaatgtcatattttgtcctctttagctagtgtgatttttgaga cataattgtaaagatttaattaagctaattggcgttctagatgaatggtcatcctttggtatttaagtaagaaaattcaaacttttaaaaatttgatttttttttttctttgaatt attttttctagtttgtactagttttctaggcgatttttttaaaaaaaatcaatggatgaatttgttttgttgcaaaataaataaataaaaaaaaaaaatatatgtagtag ggttgctggaaagattatcataaaattaaatatttaacagtttatggagaaagaagataaagaacggaagagattaacaagaaaatagttttggttttccttttaggg catttgtatcaattgaagacgaatgacaatgtttggtttggtttggtttggtagaagtaaaaaacatatagtaccgttgggcaggataagactagactacgattgga gcttaagcgagaaccatatataatatatcagtgataccattggatcgtactattgtctttgctgttgtattattgatctttatttttggttttgttttgtttaatgcaatttcaaa aatttccaaatcctttgtaattaaattgagtgtaccaatctggagaaaagatacgagactctgttgcaggtgagtctaaagtctcttgcgttcaatttcatgttcttttcta ProAtPBS3-ChIP-F3

$>>>>>>>>>>>>>>\quad$ W4

atattgaaacttcgtttgtaatttctagtctccatcactttcatcacacgactcactcaactaaataaatagtttttgttgactcccattgtagagtcttttgtggtctttgttg ProAtPBS3-ChIP-R3

W3 $\quad<<<<<<<<<<<<<<<<$

tttatgaatttaactatatttttaatcattttcttaatatttatatttgactcgcagcgtagagtcttgttgtggtctggtctttgtgtatgagttttatttttcttgcgcgaaagttt 
ProAtPBS3-ChIP-F2

$>>>>>>>>>>>>>>$

Taacttgtttcggaagtaccaaattttgcagaagttccttgtggatttctctgegtttcctgacttctcatgattcatgaaacagacaccaaaagaagaataaaaaaa ProAtPBS3-ChIP-R2

W2

$<<<<<<<<<<<<<<<<<<$

ctgatagttgcataatgttgactattaaataatcgacaagatgatacttgctgaacaattcgtcacaagttaaaacccaaggaacaaataaatcgtcaaaagttatttt aaactagattacatacgcaaattttaattttatcacaattttgcatggtactagtatactgcataattgcatttaaactttcaaaatttgaaacctaagctggtattacttgt attcctctacatattacacataatacttatttattatattaagcttaccaatgtcctttgagttattttaccaaaaaaaataatgttctttgaattagttaaactaattttccatg aaacagttagctaataaatattttccatgaagccttagatcgatgtcaaagtctcacatcatgaaaggttctcacagactctacacgtttttactaagcattgctttgg aaatttacgaaagaacattttccacgaaaccatattttcgacgaaattacattttccatgaatatgccgacaaaagaaaaacattttccatggatattagttaaacaaa ctatttatcatcagatgtctatattcctatgtaatcagctaattacttcgetcgtatattaatctattcctagttgttggaatacaaacaaaatgaagcatttgtgtgtcaca ProAtPBS3-ChIP-F1

$>>>>>>>>>>>>>>$

W1 $<<$

cttcacacgctaaggtcagtattctataatcaactaaaacattatcaaattgtgttactcggtcgagactttttatacagttaaaacattgacccagactcacgaactg

ProAtPBS3-ChIP-R1

$<<<<<<<<<<<<<<<<$

ccaaatccatttaattagttagggtttctcttgctatatatagagccaagtgttaactctgttttcatatcaactatcaactaataaccaaactatccattcttttcttcttattt tttcatatcaacttaaagagaaccaaatATG

AtPBS3-P2 tagttgcataatgttgactattaaataatcg

AtPBS3-mP2 tagttgcataatgttgaatattaaataatcg

>AtRbohD (AT5G47910) promoter: contains 5 canonical W-boxes, $2.4 \mathrm{~kb}$

Atataagcaaagccttttgtcgaaaatacacatgttggcaagttgtgggagccaccaacataagcatattatattcaaacaaatgttcaatcgcacatgtgatggtt tagcgatttagctaccgcacaactgaatcaaaagacttgttaaattgctctcttagtcttatcataccttcatctcatatccattataatcatctttagcaatactattttatt tttaactacgaaaaataaaccttgttacgecgttattaacagtgatcattttttcgttaaagaaatttaacattcaaaaaagataaagaatgatcaccaataagcgtca

ProAtRbohD-ChIP-F4

$>>>>>>>>>>>>>$

tgatcttccgtatggtgatgataaacatcgtttgcagttttgttgtatagtacaacgtatatgtagecgtaacgtgtgaatagtgtatggttagggtcattttatttgtgaa W5

ctaccacaattttgatagcagtggagataagtcaaaggttcgtcttctaattaattaacagcgattattagactatctaacttttcttcttttcttattttgataataactatat ProAtRbohD-ChIP-R4

$<<<<<<<<<<<<<<$

Atcttctttagccaccttactttctcatcgggttctatttaaatagggacaaagaaaacaaaccatcgatttgtactatgtgctcaattatatcatatgtttgtctccatct atgtatctatttctcttttccatttggtaaattatttaagaccttaaactgacttttctcgtgggeggcgtcgaagttttggttaacagacaaaacatctgttcatgatgc ctgcataactctatgttttatactactcatttgccctctatttcatgctactcttaaaaatagctaaataaaaatacttcaatattagaaaaaaatcttcttgatgtcaaaa atacactttagatatcccctctagttcttgtgatgctaatatattacaaaattgtttatatatatagaaatattacctctagcaatacaaaaatcttgttatatcaattaaatat aacactatccgactagtgtttactaaaataacacataatttttaattatgatctttgaattttagtttatgtattacgctttataattttgttatcaaaaactataaaagctcaa aagtgagcatctataccctaattccgtttttatgcatacacttaggtttatttttattacgagaatctgtgtttagtatatataaaatagagtttatgaatgactaagttaaat acttcaatactaattaaagtaagtcgtattgatgcagacaatataaactttagatatctccgctagtttgtataatgctaatagattcaaattgtctttatgtagaggaatt acctcaaataacataaaattttcattttattaattaaatctcattttgtcatttcaaaagtcattttaattactgtgttatttgtctaaagtatactccaaacaagaaaccaaa

$>>>>>$

gtagaaggtcagtgcccaagagacaaataaacttaagcatgtccactctttttagcgatcggatcgettatctgttcttatccattatggaacatgaacgatgttcett ProAtRbohD-ChIP-F3

$>>>>>>>>$ W4

taagactaccacattcaaacgaaaaataagtcatcgatgatagactggatagttatagtttgatgatctagctaattgactttcatatttacataaaacagaaatgtgtt

ProAtRbohD-ChIP-R3

$>>>>>>>>>>>>>>>$

tttatcaaaaaagaaaaaagaaaaaacagaaaatgaacaatacatctcacatggagtttgtacaagaatccataataattttttgttcggtacagtaggttagatata ProAtRbohD-ChIP-F2

$<<<<<<<<<<<<<<<<$

W3

catttgaatacagtttacaagccaaatgttagaagcaacatatttatatagtatcatcaatatacgatccaaaatgaattatagattgacttatggaaaagttagatact ProAtRbohD-ChIP-R2

$<<<<<<<<<<<<<<$

atatgcatgattgccaaatacattgaaaaataacaatttaagttcttcaaacaaaagtttataaatgactcggtgtttgacataactcggggttgtttttctttgttaattc

ProAtRbohD-ChIP-F1

$<<<<<<<<<<<<<<$

acacacggggttggtgtataaatgacttcggtgtttgaaatgtatgacacggtataaataaaaaaggtaaggaagaaatgaagaatagaagaagaacaaaaaa 

aagaatggcaatttgcatgccatttatttcatctattcctccttcactctataaatatattttaaacaaaatcaatttcttccatacacaaaaatcaaacaccttttgagag cggttatttttctctatcaactaatacagtaaccttacgggtgtttatttgtatagatctctgtggtttcttggccaaatctagtgagatctttttggtttctcgaattcg $\mathbf{A}$ TG

AtRbohD-P1 aaaaagtcaaaataaatggtcagtaaaggattttgaccagacc

AtRbohD-mP1 aaaaatccaaaataaatggtcagtaaaggattttggacagacc

AtRbohD-P2 aatgaattatagattgacttatggaaaagt

AtRbohD-mP2 aatgaattatagattgaattatggaaaagt

AtRbohD-P3 tgatctagctaattgactttcatatttaca

AtRbohD-mP3 tgatctagctaattgaatttcatatttaca

>AtSEN4 (AT4G30270) promoter: contains 3 canonical W-boxes, $1.3 \mathrm{~kb}$

ProAtSEN4-W2-ChIP-F (W3)

$>>>>>>>>>>>>>>>>>>>>>$

Ggttgcacatattgattagtgattaatatagttttgtagtattttgcaatgaaacatcttaattgactcccatcattaattagtgaccaaccaaaagaaattgaccttttga ProAtSEN4-W2-ChIP-R

$<<<<<<<<<<<<<<<<<<$

tttagaagaaagagaaggaaagcgtgtgtgtggttacttgcttgcaactttgctaacgcaatcaaatcttttaacccatacactatactcatacttttcttcgttgcatct attctcatttcatttcacacaataatatggagtacaatattattttaatcacatcgatcatagattcatgataatattgccaaacaaaatgtaagcttaaaaacttttaatgt W1

taaaatgtccaaagaaaagctcgacgtgagtcaaagcaagttgtttttattccaaaaccatggtccagttacgaccccataataggatacatacaaacttgaaaag ttataaaaaaattcaagaaaaaagatattcacaagacatattctcttcatatttcaaccaaattcgagaatttgaagtcgaacaattccacacgtaatatccctctag acctctggtttcttctttccttcctataggtctcgccgtagtgatctaagctttatgaaaacatttgaatttcgagattcgtgtaaatacataaataaataacaaaagatg caaccaattatatagcaaacgatattattaataaagattattaaattataacacaccaagaaaaattattaaagtatatcctagcatttactaatagaagagtctaatttt aatttgttattgctcaaacacagtatataattttagagatttctttggtttacgtcaaaaaagtctgtctcaattttaatattttagtcggtcatcagtatcgtccaaacaat cattttacttttgttatgataaaatacggaattcctccctaatgacaatcaaattcgaattcaaaagagctaaacaacatatttctaacaaataatatgctcatgcatgc taatatttactgtaacatttaaaaaattaaggatggtaaacataatctgettaccaaatgatggagatactcaagagtccagctgtaatacatcttgcatgcaatatct tgatattagcctccttttgttcacccactctcttctcttcttcttctcatttatttatatgttaaactctctcccactatatatatctctccccettcttctctcttcctcacattcct caccaaaccetctccaaaacacacccacacgtacgcacacacacaaagacaATG

\section{AtSEN4-P2}

Aattgactcccatcattaattagtgaccaaccaaaagaaattgaccttttgatttagaagaaagagaaggaaagcgtgtgtgtggttacttgcttgcaactttgcta acgcaatcaaatcttttaaccca

>AtSAG14 (AT5G20230) promoter: contains 1 canonical W-box, 644 bp

Gcaagtatatatacttgttgggttgatttttcttgaatacgacctgaattggtacaacggacccatgcaaagaaagacaatcgagcctagaaatttctgaacaattat cgacttatccatataaatgctataaacacctaatatctcatccatatccacatttaacactttgtttttctaacattagtatgctactattcttcgatgccttaatatcggat

AtSAG14-W1-ChIP-F

$>>>>>>>$

ggttattattaggtagttatatttagtctgaacactaaattttataatcattacccaaaaagtaattagtcacgttttttacttgatctaaatttacaaatgtacaatgtcttta

AtSAG14-W1-ChIP-R

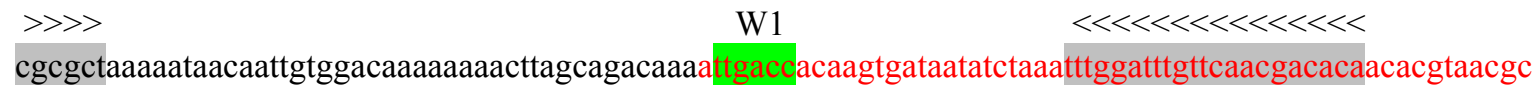
gtcttcc gtatcttgcgtatgactccttgagagcctctctttaattgtcctataaatataagtctctcttgcaagacttttgaaaccacaacaaacacaaaaatcaaaa ggggtcatttactatcttttaagagacgacatatatagttctcgaccaagaaaagaaaagggggtgacctgagttcttcaacacataacataATG

>AtActin 2 (ACT2, AT3G18780): does not contain any W-box, $2.1 \mathrm{~kb}$

tgaaaactttataatataaaaaacatgaacgtggtctggectgetttgcataaaccaacccatataataaatagtgagaaaattaaatgtaatgataatagtagcaat gtataaatcaagaagtagtttcgtaatgatggacacaacgtttcgtaggtgccagcttcatcaccttcccacattctctctctttctcacctggtacgatcgtaaccg gcacgtgcgattcacgtggctcgcaaaaaagttgactttttagctttgatttaatctcaacaaacattaaaaagtttatgatgatgaatgattagtttgctttttcttttgta 
taacatgagaggttacatgttattgctcatatctttcacttacatagcgcatcgcacgatgatgatttgatctgatatacggtctgatggtcatatgttcagtttttaagct aactagatgagttcaaatacataaaatcataatatatgcgtatagttaaatatattcaccatagaatagctagctaactaatacatggatcgatgccaaaagtccca aaactatataagtgacgaggacaccaacaaactattcgtcacgtcgacaaaatttagaacgaacttaattatgatctcaaatacattgatacatatctcatctagatc taggttatcattatgtaagaaagttttgacgaatatggcacgacaaaatggctagactcgatgtaattggtatctcaactcaacattatacttataccaaacattagtta gacaaaatttaaacaactattttttatgtatgcaagagtcagcatatgtataattgattcagaatcgttttgacgagttcggatgtagtagtagccattatttaatgtacat actaatcgtgaatagtgaatatgatgaaacattgtatcttattgtataaatatccataaacacatcatgaaagacactttctttcacggtctgaattaattatgatacaatt ctaatagaaaacgaattaaattacgttgaattgtatgaaatctaattgaacaagccaaccacgacgacgactaacgttgcetggattgactcggtttaagttaacca ctaaaaaaacggagctgtcatgtaacacgcggatcgagcaggtcacagtcatgaagccatcaaagcaaaagaactaatccaagggctgagatgattaattagt ttaaaaattagttaacacgagggaaaaggctgtctgacagccaggtcacgttatctttacctgtggtcgaaatgattcgtgtctgtcgattttaattattttttgaaag gccgaaaataaagttgtaagagataaaccegcctatataaattcatatatttcctctccgctttgaattgtctcgttgtcctcctcactttcatcagccgttttgaatctc cggcgacttgacagagaagaacaaggaagaagactaagagagaaagtaagagataatccaggagattcattctccgttttgaatcttcctcaatctcatcttcttc ProACT2-ChIP-F >>>>>>>>>>>>>

cgetctttctttccaaggtaataggaactttctggatctactttatttgctggatctcgatcttgttttctcaatttccttgagatctggaattcgtttaatttggatctgtgaa ProACT2-ChIP-R

$<<<<<<<<<<<<<<$

cctccactaaatcttttggttttactagaatcgatctaagttgaccgatcagttagctcgattatagctaccagaatttggcttgaccttgatggagagatccatgttca tgttacctgggaaatgatttgtatatgtgaattgaaatctgaactgttgaagttagattgaatctgaacactgtcaatgttagattgaatctgaacactgtttaaggttag atgaagtttgtgtatagattcttcgaaactttaggatttgtagtgtcgtacgttgaacagaaagctatttctgattcaatcagggtttatttgactgtattgaactctttttgt gtgtttgcagctcataaaaaATG

> BnaICS1 (BnaC06g22820D) promoter: contains 4 canonical W-boxes, $2.3 \mathrm{~kb}$ cattgtcagacgcgtgatttcgtgagcttgtctagcttctcaaacttcgtttaaaatttccaactatagcatgcgacgttgaaatgcaacaaccattccaagtcataaa tgatagaaacaaaatgtgatcaccagtgaccacgtccaacatttcatgatctataaagtcacactataatacaatatcgtaggactagaccgtgtgtagttgcag BnaICS1-ChIP-F2

$>>>>>>>>>>>>>$

tttctttagattaacatccgccaatggtagttgagttatggtttggtttcattatattcttatctgaatgacttgctaatttttaataggtcgtcatgataaataacaatttga BnaICS1-ChIP-R2

W3 W2 $<<<<<<<<<<<<<<<<$

cttttaaagtcaagtcacttataatctaataaatatatttgttttgaccacgatgattcaatttgacagaaaaaatataataggtctatcaaatagctggtgagccatc

gtaatcatcctatggactttggactaaaatgtgtgtcttagttatttcagggaaattttggggaaattgctacaaactcctatactattcagtctcctctcactcttgacg acattatgatttattcagaagtgaaaatgaatctttgtattttcagtgtgttttcgttttacattaaaaatgtttgaataatttataaccgggacgtgaattgatgatttttca

BnaICS1-ChIP-F2

>>>>>>>>>>>>>> W1

aagagccactttcctcgcaaagaagatggttagactgaattgactccttaaataatatatgggattatacagataacaatagatggatttataacttcgttgtagaaa BnaICS1-ChIP-R2

$<<<<<<<<<<<<<<$

caaggtggatatttgtagattggattcatcatttgcaaagtagaaaaatggaaaaatattaaagattggtgtcaaagtaagaaaatatgagaaaatgtgtccatgct gtaattaatctagctaaaatatgataaacaaggcacataagaatttaatctaagataaatcttggttttaacttttattgggatgacatgetgttggaatatcttttgtctgt tttggggtcgattttaactatgaccacatatttttgattaattatatcaattattctagagggatccgagtctgtcaattaagatcagaatctttcatattggttatgcttttc attttgtttgaaccgttgatttatgtcttaatttcagttgatttcatattgtttctatctttcatagctcagcattacactaaaaagtttcattaatcatatcctaatttacaaaa cgacagaacaagtgcaacgaaatacagctattactaccatcatatccatcacatgtggtcagtcagtagacgaacagatttggagtcgetggagacgtctgagg caagtaggctaaagaaaaggaagctataagttatacaatgggctggctattttgatggttttagatggttcgagacccatcatctaaacattcgcgatgacgtggc agtttgattggtagagaatattttatcctatgtggcattcctaagaagcttcaaaattagtagcttttatatagtaggatatttatttcttctcatctcgaacaacattgtgtg cttattcatgtatatactttttatgttacgtaaaccacaatttattctaattcagtttatacttccatacttcatagtaatagaccaaccaaaaacaagtttctttctttctttag attaagaatattaaattgttatgtgaataaataaagatataacgaaagaaaagtatgatccaatttcaagtgaagaaattaaaataaaagaaaatttctgagtagttta cttagcaattctgagaaatttaagtgtgaaaatgaaatgaattcttcaattttaaaatggggcctagctacacccgtcccetatttattgagggcgaatccataatcca tatcatcaaatatctacttgaaccaaagagaaaaactatatagtatattcctccaaacagaaaaaaagaaataaacaaaatatataaacaaatcactcaccatctct cttttgttcgttctctaagcaatctccaATG

>BnaPBS3 (BnaA10g19970D) promoter: contains 2 canonical W-boxes, $1.9 \mathrm{~kb}$

Agagacttacagtagaagatggagaaggetttacctaacccacgtgaccacgtcagaccagactaaactgaaagtcattaagaaatagaacctaaaatcttgtt gctaattaaatagtgactttcaacactgaaaattacttggaatctggttttctttttccagagtgtttctcagcaatatccctcaaggtccaacttgataaagtccacta W2

Ttttaaggctttagtttgaaggtcaaaatcatttctattaaatattttgacttctaagttctaacagatcgattaaatgttgggaacatgatcaaacatcagtttctaaatc atttgttactaaatttaaattattctgaataaataaataatatataaatatcactataagtattttatatttctatatttaaatttaaattattctgaataaatcattttatatttgtca 
acaaaaattaaaacttaggtttaagaaaaatggaatagagttagtggaatgggaaaatatattcatagccatgaatgtggaaaatagacattattaatatgagaa aaggacaataattaatttatttgtttttcctttcttggactaaaaacgcattagtaccagttgaagataatttaacaatatttcgtttttttgtttaaacaaaaaaacatagt actgttaggtcggataagactacgattggagatatcgtgagaaccatataatatcactctttcgtactgcgetattgtgtttcttgatattttttattttttacatttgtttgaa ttaatacattttcacaattttctgctccattgtgtgtgaatcaacatggagaagagagaatagtctactccaggtgagtttaaagtcctgcgtaagtttcgtttgcttttct gattatctttacttgtttcaacaaaataaaaaatttaaaactatacttatcacagaaacaacaaggtttccgattgaatattcttgtattgaaacttcgtttgtaatttctag

W1

accttcgtcacgactcacacgaatcccagctaaatagttttgtattggttgaccaacaaaaaatatataaagtgaactatcaatggggtctttgtgtatgaatattatt ttcttcaacagaagtttcaacttaatttggaagaacaatgcataaagaaatttcttgtcgattttccettaatttctccacgttttcetgtgttgatccttatattttattccac aagtcgcagaatgttgacggtcgaatacttggctacatgataaatggctaccaaaaacttatctcaaattattttaaataaataaatactttcgtaattttacaagcgtg gatatgtcaaagtgtcaaaccaaactgaaaaatcaaaatttattgaattcttttaataaaaaatatcaactaaacatatgtatatgacaaaaaacatatgtgaaaca ccttctaacgattgatcttaagaattgtcgetgaccatgaattctcagatgectaagattcgtatcattaacgttcattccacattgttaagtctatacaatggtccttaat tagaacccccaggaattagttaagctgtatctttccatgtaacttcaattatattgtctttgacaaaatcagtttcttgtcatattcctgaagtttctatatcattactcttctc atacactacacgtacgtatataatatggcatggaatcgttcacacggtgaggtctaagtcgtctaatcaattaaacatatcgatcaagttgtgcttttcacttacgactt ttctatagttaatcatctaaacaatgaccccaaacttccaatacttttgctatatataggacttaagcattcactttttatcatatcatcaacaaaaaatcatacccaacaa aaaaacattcactctctatcttcatatcaattagagagaaccaaaaATG

>BnaRbohD (BnaC07g26270D) promoter: contains 3 canonical W-boxes, $1.2 \mathrm{~kb}$

Gcaaacttttaatataccttttcaacggttcttaaaatgatatttatgaggaatcatcaatactggatttaaataccggttatccggacaaaggatattgtgccatgac

ProBnaRbohD-ChIP-F3

$>>>>>>>>>>>>>>>>>>>$

gtctgtattctacataacatgatgtagctagctttcctattacatgtcgctcatgaggttcacgcgtttggetaagtagatgtcaatatgtactaagtacgttattatcgg

ProBnaRbohD-ChIP-R3

$<<<<<<<<<<<<<<$

Gagttatgectgaatgaactatcatgtgcgtatcagcgtatggtttgttagtttttttggccettggtagacttcaaaatatgcttgacacactgtgcaggagagtgtg tgtctttcatagtttacggatccaaaattcacctgttgcaccaggattggaaatgataaatagataacggaatagatgggaattagttctaacatctatagtactgtat ProBnaRbohD-ChIP-F2 (W3)

$>>>>>>>>>>>>>>>>>>$

atggcaattgactattttagtatataaacaaacaaagaaatcaaaatagatgcatctcacatggaggtgtacatcaatgatcagattagccaggctataacactagt ProBnaRbohD-ChIP-R2

$<<<<<<<<<<<<<<<<<<<<<$

taaggtatgtattgaaaacaaattacaattagtcaaatgttggaggcaacatatttatatggtcccatcatcatagctattactttattttatgtaattgtcaaacaaaga ggattatattacaagtttagacaaacctaagtaaatcatccgctactaaaaattgaagtctctcttttcctctccgcaaattcacacgggtttgttatatatagatgcatg ProBnaRbohD-ChIP-F1

>>>>>>>>>>>>>>>>>>>

aatgaacttgaaggcaaaccataaatgactcggtgttgaaatgtataatgtgactcggtggaaatagaaacagagaagaatagaaatgtagacaaaataaaatta ProBnaRbohD-ChIP-R1

W2

W1 $<<<<<<<<<<<<<<$

aattaaaacaacgaaaaaaagtcaaaataaatggtcagtaaagggtttgaccggaccggagcggtccatccatagtttcccactaccttcctggattatgaaattg caatttgcatattgcatattgcatgcccattcatgtcatctgtccctcattttctctatatatatattttatgcaaaaatcaatttcttccatagaccaaaaaaatcaaacac cttttaggagaagagatttctttctcgacctaatatagtaacccacaattattgttatcttagccaaaatcaagtgattatctctttttttcatcctttgaaacaaaATG

>BnaRbohF (BnaA09g11870D) promoter: does not contain any canonical W-box, $2.0 \mathrm{~kb}$ tttattgatgaatagatgtatcatgtatgaaactgctggaataaaagaaggtttcattgtgtttttctaacgtgtgatatactaatctatcattaatcatgcttcagtaaata accaattatacgataagtgggctcgttatattgaataaccagtggcggatgaaaaaaatttagttgggggtacaatactaaaaacaacagaaacaaaattgaattg gaggcacttttgttgtttattcagtaaactaaaagaattttaatcaaattaacatagaacattcaaaggaaaaaaaaacagttgggtgtacgtgactcccttaccgta ggcgtccgtccgccccgagaataacacgatattttaatatcaggttcaggtatagtacagtcaaaatcttcatcaaaaaaattgtttttgacaaaagatcttcatca aattttcacatttaactttcatacttgcatggagctttttgtttttattttacaactttaacacagcctaatatttgaattgaaatctctatgaaaaaaatgaagaggcgcac acaaccactcgagatataaattggcatgtgtgccttcctataaataatatattggtaagcaactccgtgtcttatgtgagtatccgcgatacaagattattcgacgac ccatgaaacatactcagtttgatgtaaaatattttgaatcactttaaaaatgctgttatcaaaattagtttgctcttcttaaactaaactcgtttcagttatgagaatatattt gtcgtcgtcgtggacttgacgatatactacatcaatcactacaacacttctatccaaaagtttcgtccaagcttttattcaaagtcgtccettttgttttctctcagagat ttacaagcacaactttggcaaaaacgcgtcaggcgaaatagacttaagtagacttaacagagagacaaattacattcacacagtcacttctcacttgtgtaaaacg ccatggatacagtagtatacatgtcttacatacttcataaaagagcaaactattagtgatatttacggataaacttgaaaaaggaaaagataaattctccaaaatagc actagtttttttgtcccagaccttaaacctaacaccaaatttagaattctaattaaactgtataatttcagtatcaaaagaataaaaattcataataaaaaggaaatttaa accctgaatttaaaacgtagaacccaaacataatatttaactttctttgtagtttgatttaggagcaacatgtatttttacaaaataatttgttctattttgcaaattgtaaat ttgatgtgctcaagtttagaaggagaataaaaaaaattctggtaaacaaaatatagtttaatatttttgtcaatcaataacaaaataaaattataaaaagataaaaacat taaaatcaaaccggacgctcgtggtcttgtggtaaacagttaacagctgtgagtttcattgcctcttagatattttcggcagatttccggcttaactccggtagacg gtcattaggcggtagtcagatctctttcgaggacattcggataaaacattaaaaatcaatgttattattgtaaagtttttttttttcaaaaagagtatattgcgcaaa ctttttgccggtccagttcgaacctcatgggecgtgtttcctctctcttcgtcaacgttctgcttcagtgcgtttctctctttctctctctctagtctttttacctctgcatca 
ctttctctctctctctctccctcgcctataaagcacagatatgcacagcgcgtgaaaaatggctttctccactgagccacgacgegtttgagatctctctctctccet ctctctctttccetctcetcetttcaaagattccacctaccaatatatatactactgtatatgetacgtgtatattcatcettcceccaacagtttcatccetagtgttcgec ggatctttccATG

>BnaSEN4 (BnaC01g08130D) promoter: does not contain any canonical W-box, $2.1 \mathrm{~kb}$

ttatagtctgtcttaatacccattaatctagttgcatatgataaggttgaatgaccatcactacaattttgatacaaaggctattttcatgcatccaacatgtcaaaaaat ctcatagatttgggatttggttcttccctctataatgatcatgtaccatttgctcagtacctacaccataatctatatccgttctagattcttctaatctaatatcaggctg aagttcactaacaggctgaggttcgctagtactaccatattcataaccagtttccccatgaaggtaccaaactttataattacgtgaaaacctttcatatacaaatga gtccaaacatcaaattcttttataaccttattattattgcaagtagagcagagacattttaacataccacttttgcatccggttgctgttgaacaagcctcatgaattctc caatccettgaacgtattctttcgtaagcaaattggtgttgggatccaaatgaggtttatccatccacgaacgataataagctcctgaagacatgattttcacggaatt gctatgactaaagagaattaagagagaatgaagtgtgaatgagttgaatgaggaggggttgcatttataggaaattgcttacggccatccgatgactttccgatg gatgtaaagcagtccgtcggaataccgtcggtattttccaatctcaaacggctatacaacggtcatatatatttgtcggcaacggtcacatggttcgtcggaaattc gtcagaaaattccgacggaataccgacgacggtaacggttatatctgttaatcggaatgtcgtcggaagtttatcggtatattccgacgaatttccgacgactaca acagttacatgttttatcggaatgtcgtcggaaaattccaacgagccatgcttcgtcggaattctgtcggaaatggccgacggaattccgacgacttcaaatttttg gttttcatcggaaattggtcaaaaatccgtcacaaacgtccgacgacattgaagtccgtcggaacctccgtcagaattcggcgtgttttcttgtagtgatgcatgta gaaagttcgacttgagggtcgaagcaagttttattcaaaaaatatggtaaagttaacgacccagtaattggatacatacaaactcttaaaactacaaaaaaaaa agaatatcaaaatgtatatattccaagacaaaagatatgccaattatacattgtcctcaaatttcaactaaattcgaagtcgaacaaccacacgtaatatccetctag acctcttatttctctttcctcctcccactatacaggectctacgtggtaatgaagatatacaaataattttgaatttggaaattcgtgtaaaatactctctccgttttaaata atttttttgttacaaaataagtgtcgttttcgattttcaatgcaaaatttattaattttatgtaaattttatttttctattgattgaaatatggttaggtgtataggtaatagtgtct ttttacaggaaatgtacaatattaattgttttcttaatccgtgtgccgaaacctctaaaacgacaaacacactaagtatctacgaagcatgcaaccaattatatagcaa acgttatttaaacaattcttaaagctaaaactcaaaactggcaaacagagaaaaaataaagaaagtactcaagtactgttaaccaaagggaaagttaatactagca ttatcaatacacgactttaaagaaaagcagatatcaactcataatttgtaatcttctcaaacatacttttaagaatgaaacaactcgtttctaacagtatgaccatgttt atatttcaattaaggttggtaaaataatctgctcaccaaatgatggaagtaatcgagagtccagctgtaatacatcttgcatgcaatgtcetgatatttagectcttttgt tcacccactctcctcttaacattacttattattctccacccactatatatatctctctccettcttctctcttcctcacattcctcaccaaactccaaacacatacacccac acgtacgcacacataacaATG

>BnaSAG14 (BnaA10g15190D) promoter: contains 2 canonical W-boxes, 885 bp

Tatatgtataaaatttaacgattaatccatgtacaatttacctatgtcgacatatagtttataaataataaacacacctaatatttatttttgaaaataaataaatacacct

W2

aatattacaacaaaatacactgacctttgaccctttaaccatttttcataacaatagaacatttctaactcatctctatatttttcttgtaaaatatatatttatatgatcagct cacttccttttttaaagaaaatagtatatttctgtatttacagtaataaatagcattttctatttgaaaaatatacattgaagaagtctcttgttctattttataattcttgtatct BnaSAG14-ChIP-F2

>>>>>>>>>>>>>>

tttagaaaaaaaagtatagaggtagagtgaagatgttcttagcattctgtaagccetctccatttgttgtttattttaccattattattagtatgttacatacatgtagta BnaSAG14-ChIP-R2/BnaSAG14-ChIP-F1

$<<<<<<<<<<<<<<<<<$

cgaacaaaagatttataatcaaaagacaaaagtaaatagtaaatagtcacgttttcttacttgatcaacgtgtagggatggacaatactttacgcgctaaaaataac W1

aaacaaattttctggaaaaaaagagaaactaagcaggcaaatattgaccaaaggtggtggtgataatatccaatatgaatttattcaacgactaccaaacacgtat BnaSAG14-ChIP-R1

$<<<<<<<<<<<<<<<<$

acgcgtcttcgcatatcttgcgttaatgacccttgaaaacctctctttctccttataaataactctctcctttgagactttctgaaccataacaaacacaaaaatcgaa aagggtcttaaatacttatcttttaagacaacgtttatcaagttatcgaccaagaaaaagaaaaaaaagggggtgacetgagtttttcaacacaacacaaagcc $\mathrm{A}$ TG

Fig. S16 Sequence analysis of the promoter regions of putative targets of BnaWSR1. Canonical W-box

(T)TGACC/T elements are highlighted in green color, while non-canonical W-box in yellow. 5' untranslated

region (UTR) of each gene is highlighted in red letters. Primers are highlighted in grey background. 
A
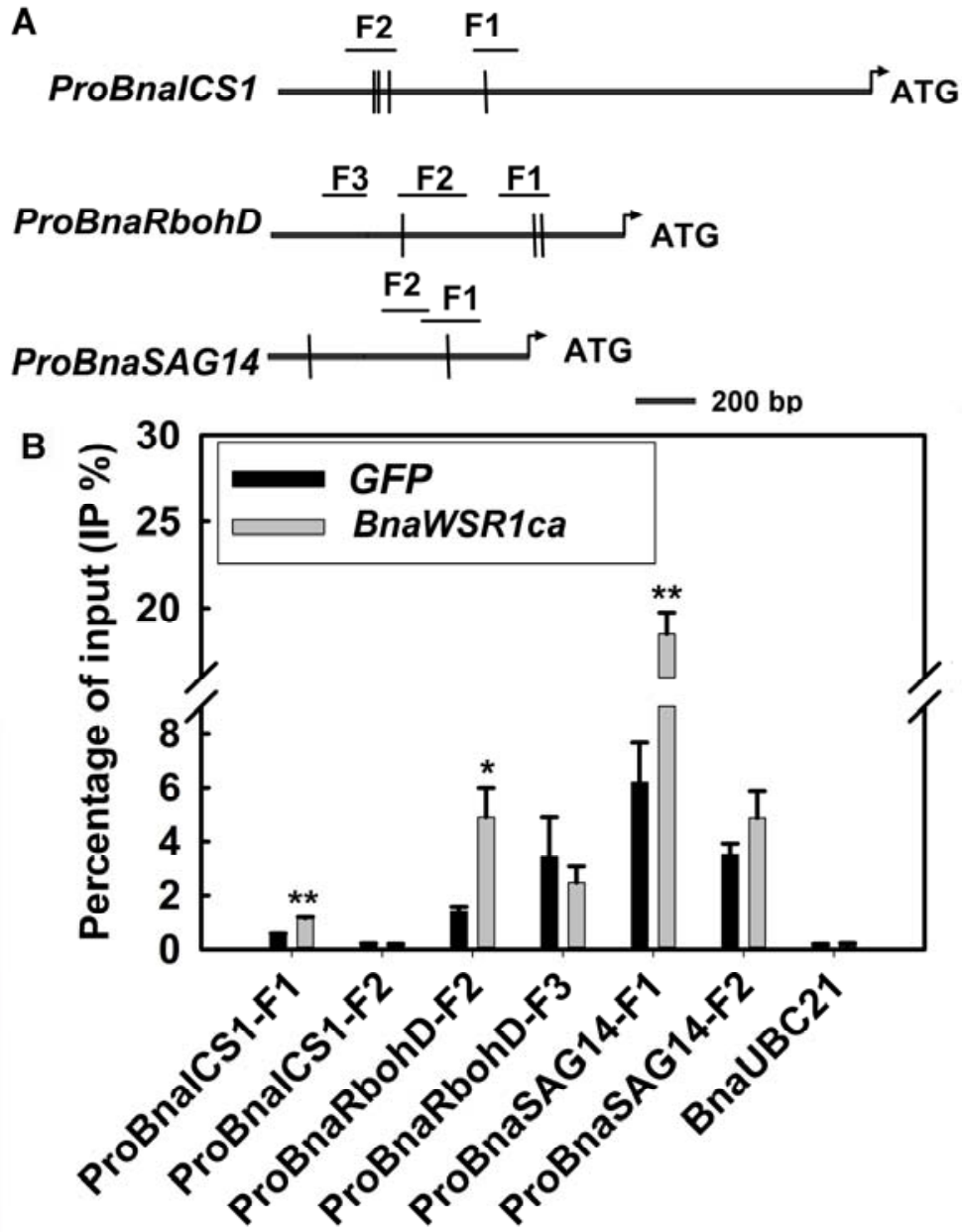

Fig. S17 The ChIP-qPCR assay of binding of BnaWSR1 to relevant promoters of rapeseed genes. (A) Schematic diagrams of the promoter regions of BnaICS1, BnaRbohD, and BnaSAG14 as well as the Ubiquitin 21 (UBC21) control gene. W-box cis-elements were depicted by vertical lines. The prefix 'F' indicates the fragment detected by qPCR following ChIP, which used chromatin prepared from BnaWSR1ca-HA plants using an anti-HA antibody. The promoter region of UBC21 was amplified as a control. (B) ChIP-qPCR results. The enrichment was presented as a percentage of input DNA. Data are means \pm SE of three replicates. Asterisks denote significant differences in enrichment $(* P<0.05 ; * * P<0.01)$ compared to the GFP control by Student's $t$-test. 


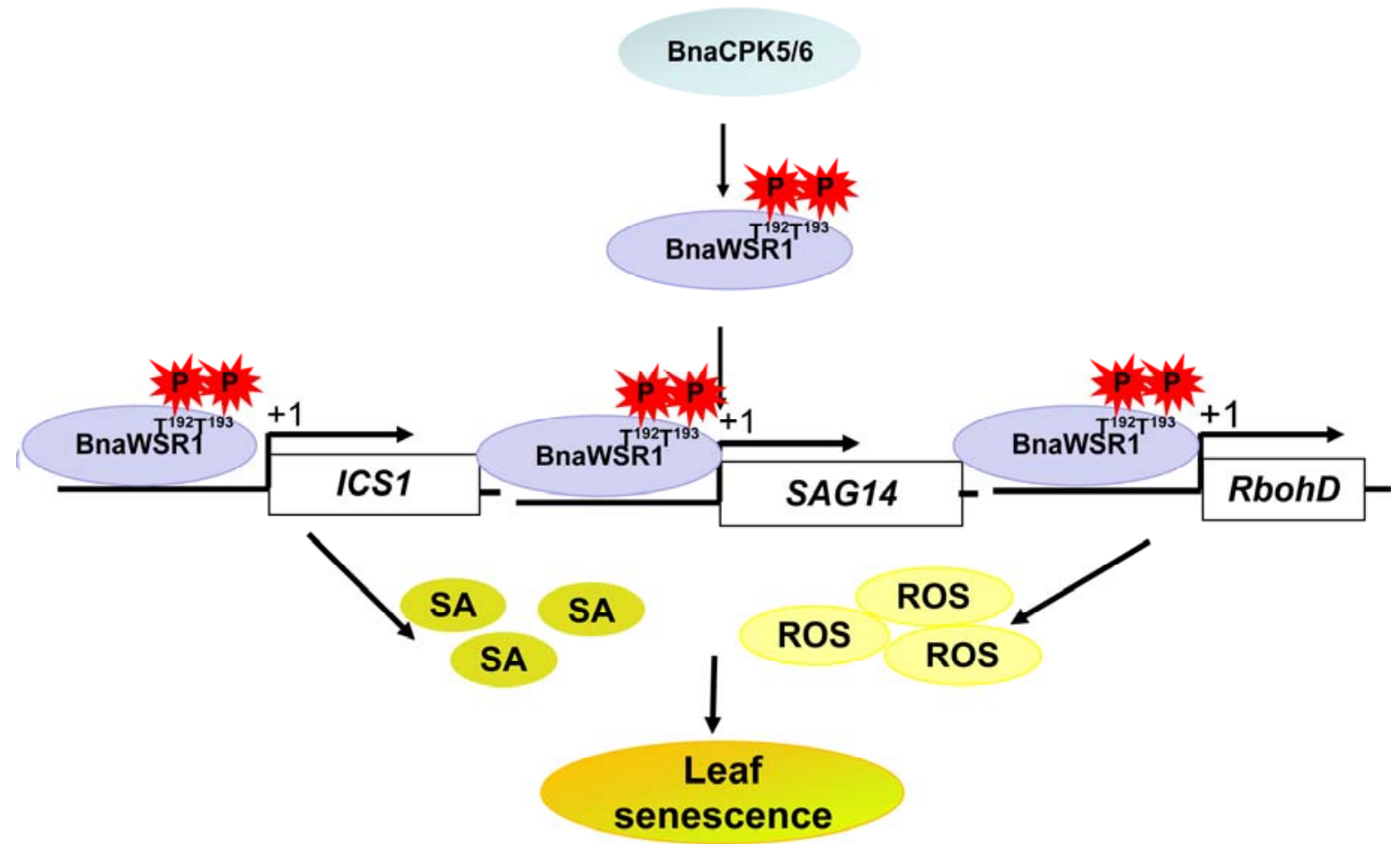

Fig. S18 A proposed model illustrating BnaWSR1 involved in ROS- and SA-mediated leaf senescence.

BnaCPK5 and BnaCPK6 can phosphorylate the Threonine (T) 192 and T193 of BnaWSR1. Phosphorylated BnaWSR1 binds directly and specifically to cis-element W-box in the promoters of ICS1, RbohD and SAG14, encoded proteins of which regulate the transcription of those genes involved in SA and ROS synthesis or cell wall degradation and PCD processes. As a result, this eventually leads to leaf senescence.

\section{Supporting Experimental procedures}

\section{Hormonal treatments and pathogen inoculation}

7-d-old seedlings of rapeseed were cultured on half strength MS (1/2 MS) supplemented with 2 mM salicylic acid (SA), $100 \mu \mathrm{M}$ jasmonic acid (JA) (Sigma), $50 \mu \mathrm{M}$ abscisic acid (ABA) (土-ABA, Sigma), $10 \mu \mathrm{M}$ methyl viologen (MV) (Sigma), $25 \mu \mathrm{M}$ 1-aminocyclopropane-1-carboxylic acid (ACC) (Sigma), $200 \mathrm{mM} \mathrm{NaCl}$ (MP Biomedicals). For cold treatment, seedlings were grown on $1 / 2 \mathrm{MS}$ medium at $4^{\circ} \mathrm{C}$. Seedling growing on $1 / 2$ 
MS medium without any treatment was set up as the control. Samples were harvested at $1 \mathrm{~h}, 6 \mathrm{~h} \mathrm{and} 24 \mathrm{~h}$ after treatments. Fungal inoculation was followed as described before ${ }^{1}$. For Sclerotinia scleoriotum (SX09904, kindly provided by prof. Lili Huang, Northwest A \& F University) treatment, mycelia front edge grown on potato dextrose agar (PDA) agar at $2.5 \mathrm{~d}$ were taken to inoculate the $1^{\text {st }}$ and $2^{\text {nd }}$ true leaves of 18 -d-old rapeseed plants growing in soil and kept in humidity chamber with a relative humidity of $100 \%$ for $24 \mathrm{~h}$. Samples were then harvested at $1 \mathrm{~h}, 12 \mathrm{~h}$ and $24 \mathrm{~h}$. Three biological replicates were performed for each treatment.

\section{Subcellular localization assay}

To examine the localization of BnaWSR1 in planta, the N-terminal GFP fusion of BnaWSR1 was transiently expressed in tobacco leaves. The nucleus was indicated by nuclear localization signal (NLS)-fused mCherry. Fluorescence signals were examined 2 d later under a confocal microscope (FV1000MPE, Japan).

\section{Transcriptional activity assay}

The coding region of BnaWSR1 was subcloned into the binary vector pYJHA, and was used as the effector. The reporter plasmid contains five tandem repeats of W-box element fused with the minimal TATA region of CaMV35S promoter, which were located upstream of the Firefly Luciferase (LUC) gene in the pGreenII0800LUC vector ${ }^{2}$. The internal control is Renilla Luciferase (REN) driven by $35 \mathrm{~S}$ promoter. The control effector plasmid is similar to the above effector, except that BnaWSR1 was replaced with GFP. Plasmids were transformed into agrobacterium strain GV3101 (pSoup) individually. Agrobacterial cultures transformed with one of the two effector plasmids and the reporter plasmid $(9: 1, \mathrm{v} / \mathrm{v})$ were co-infiltrated into the lower epidermal side of $30 \mathrm{~d}$ old tobacco leaves. At 2 and 3 dpi, leaf discs were harvested and stored in a $-80^{\circ} \mathrm{C}$ ultrafreezer. LUC and REN activities were quantified by a dual luciferase assay kit according to the manufacturer's instructions (Promega) on a GloMax ${ }^{\mathrm{TM} 20-20}$ luminometer (Promega). Three biological replicates were conducted for this assay.

\section{Expression of BnaWSR1 and BnaWSR1ca in tobacco}

BnaWSR1 and BnaWSR1ca were introduced into plant expression vector pYJHA and expressed in tobacco leaves through agrobacterium-mediated infiltration ${ }^{3}$. Leaves were detached at 66 hours post infiltration (hpi) 
and 4,5 and 6 days post infiltration (dpi) for detection of hydrogen peroxide $\left(\mathrm{H}_{2} \mathrm{O}_{2}\right)$ using 3,3'diaminobenzidine (DAB) (MP Biomedicals) staining protocol previously described according to Daudi et al ${ }^{4}$. The DAB buffer contains $1 \mathrm{mg} \cdot \mathrm{mL}^{-1} \mathrm{DAB}, 0.05 \%$ Tween- 20 and $10 \mathrm{mM} \mathrm{Na} 2 \mathrm{HPO}_{4}$. Leaves were stained in the DAB buffer for $4 \mathrm{~h}$ on a shaker with a speed of 80 to $100 \mathrm{x} g$ before cleared with ethanol:acetic acid:glycerol solution ( $3: 1: 1)$.

\section{Physiological measurements}

Briefly, leaf tissues incubated in deionized water before (C1) and after (C2) boiling for 15 min were used to measure the conductivity through a DDS-307 conductivity meter (Leici, China). Relative conductivity in percentages was expressed as $(\mathrm{C} 1-\mathrm{C} 0) /(\mathrm{C} 2-\mathrm{C} 0) \times 100$ where $\mathrm{C} 0$ represented conductivity of deionized water. Leaf tissues immersed in $5 \mathrm{~mL}$ of absolute ethanol overnight at $4^{\circ} \mathrm{C}$ were used to measure absorbance at 665 , 649 and $470 \mathrm{~nm}$ on a spectrophotometer (Thermo Scientifc). Chlorophyll content was calculated using a formula of $(20.2 \times \mathrm{A} 645+8.02 \times \mathrm{A}$ A657)/g fresh weight (FW). MDA was sequentially extracted with $0.1 \%$ trichloroacetic acid (TCA, Alfa Aesar) and 2-fold volumes of 20\% TCA plus 0.5\% TBA, boiled for $30 \mathrm{~min}$. After cooling down, the absorbance was measured at 450, 532 and $600 \mathrm{~nm}$, respectively. Concentration of MDA was calculated using formula of $\mathrm{C}=6.45 \mathrm{x}\left(\mathrm{D}_{532}-\mathrm{D}_{600}\right)-0.56 \mathrm{xD}_{450}{ }^{5}$. The hydrogen peroxide content was quantified against a standard curve of $\mathrm{H}_{2} \mathrm{O}_{2}$ (Alfa Aesar). Three biological replicates were conducted for each of physiological measurements.

\section{Promoter cloning of rapeseed genes}

Promoter regions of orthologous genes from rapeseed were retrieved from the Brassica napus Genome Browser database (http://www.genoscope.cns.fr/blat-server/cgi-bin/colza/webBlat) through a Blat search using the protein sequences of orthologous Arabidopsis genes. Promoter regions (including 5'UTR) of rapeseed genes were amplified via high-fidelity Pfu DNA polymerase-mediated PCR using genomic DNA as the template with primers listed in Table S1. After restriction digestion, the PCR products were cloned into pGreenII 0800-LUC vector and sequenced.

\section{Rapeseed protoplast assay}

The coding sequence of BnaWSR1ca was amplified and subcloned into pUC19 vector. This resultant pUC19- 
BnaWSR1ca-3xHA-3xFlag plasmid was used for transfection with pUC19-GUS used as the control ${ }^{6}$. Protoplasts were isolated from 5 -week old rapeseed plants as described before ${ }^{7}$. Cell death and ROS accumulation in protoplasts were evaluated using Evans blue, fluorescein diacetate (FDA) and $\mathrm{H}_{2}$ DCFDA stainings, respectively $12 \mathrm{~h}$ after transfection as described previously ${ }^{3,8}$.

\section{ChIP-qPCR in rapeseed}

Chromatin immunoprecipitation (ChIP)-qPCR was performed using rapeseed protoplasts transfected with pUC19-BnaWSR1ca-3xHA-3xFlag according to the method described previously ${ }^{9,10}$. pUC19-GFP-3xHA$3 x$ Flag was transfected as the control. Briefly, transfected protoplasts were collected $12 \mathrm{~h}$ after transfection. Protoplasts were fixed with $1 \%$ formaldehyde in $1 \mathrm{x}$ PBS for 10 min followed with quenching by adding glycine at a working concentration of $0.1 \mathrm{M}$. The chromatin was fragmented with a tip sonicator (Ningbo Scientz, China). $150 \mu \mathrm{l}$ of sonciated chromatin was diluted ten times in a ChIP dilution buffer. After precleared using salmon sperm DNA/protein A agarose beads (Upstate, USA), 10\% input control was collected from the total $1,500 \mu \mathrm{L}$ of chromatin and the remaining $1,350 \mu \mathrm{l}$ of diluted chromatin was mixed with $3 \mu \mathrm{L}$ of anti-HA antibody (Thermo Scientific) at 1:500 for overnight and immunoprecipitated BnaWSR1ca-HA-Flag-DNA complex was captured using salmon sperm DNA/protein A agarose beads. DNA was then eluted and reverse cross-linked for qPCR assay using a CFX96 real-time system (Bio-Rad). All of the primers were designed to amplify DNA fragment ranging from 75-200 bp using Primer 3 online or PrimerSelect (DNASTAR) and primers are listed in Table S1. Specificity of all primers was examined with melting curve showing only one single peak. The percentage of input was calculated with the formula of $100 \times 2 \Delta^{(\text {(ct(input) }-\mathrm{ct}(\text { sample) } 11 .}$

\section{References}

(1) Liang, W.; Yang, B.; Yu, B.; Zhou, Z.; Li, C.; Sun, Y.; Zhang, Y.; Jia, M.; Wu, F.; Zhang, H.; Wang, B.; Deyholos, M.; Jiang, Y.-Q. Identification and analysis of MKK and MPK gene families in Canola (Brassica napus L.). BMC Genomics 2013, 14, 392.

(2) Hellens, R. P.; Allan, A. C.; Friel, E. N.; Bolitho, K.; Grafton, K.; Templeton, M. D.; Karunairetnam, S.; Gleave, A. P.; Laing, W. A. Transient expression vectors for functional genomics, quantification of promoter 
activity and RNA silencing in plants. Plant Methods 2005, 1, 13 .

(3) Yan, J.; Tong, T.; Li, X.; Chen, Q.; Dai, M.; Niu, F.; Yang, M.; Deyholos, M. K.; Yang, B.; Jiang, Y. Q. A novel NAC-type transcription factor, NAC87, from oilseed rape modulates reactive oxygen species accumulation and cell death. Plant Cell Physiol 2018, 59 (2), 290-303.

(4) Daudi, A.; Cheng, Z.; O'Brien, J. A.; Mammarella, N.; Khan, S.; Ausubel, F. M.; Bolwell, G. P. The apoplastic oxidative burst peroxidase in Arabidopsis is a major component of pattern-triggered immunity. Plant Cell 2012, 24 (1), 275-287.

(5) Jambunathan, N. Determination and detection of reactive oxygen species (ROS), lipid peroxidation, and electrolyte leakage in plants. Methods Mol Biol 2010, 639 (1), 292.

(6) Wang, B.; Guo, X.; Wang, C.; Ma, J.; Niu, F.; Zhang, H.; Yang, B.; Liang, W.; Han, F.; Jiang, Y. Q. Identification and characterization of plant-specific NAC gene family in canola (Brassica napus L.) reveal novel members involved in cell death. Plant Mol Biol 2015, 87 (4-5), 395-411.

(7) Yoo, S. D.; Cho, Y. H.; Sheen, J. Arabidopsis mesophyll protoplasts: a versatile cell system for transient gene expression analysis. Nat Protoc 2007, 2 (7), 1565-1572.

(8) Niu, F.; Wang, C.; Yan, J.; Guo, X.; Wu, F.; Yang, B.; Deyholos, M. K.; Jiang, Y. Q. Functional characterization of NAC55 transcription factor from oilseed rape (Brassica napus L.) as a novel transcriptional activator modulating reactive oxygen species accumulation and cell death. Plant Mol Biol 2016, 92, 89-104.

(9) Lee, J. H.; Jin, S.; Kim, S. Y.; Kim, W.; Ahn, J. H. A fast, efficient chromatin immunoprecipitation method for studying protein-DNA binding in Arabidopsis mesophyll protoplasts. Plant Methods 2017, 13, 42.

(10) Rehmani, M.; Chen, Q.; Yan, J.; Cui, X.; Gao, S.; Niu, F.; Yang, L.; Yang, B.; Jiang, Y.-Q. A novel stressresponsive BnaNAL1 transcriptional activator in oilseed rape positively modulates reactive oxygen species production and cell death. Environ Exp Bot. 2019, 163, 1-14.

(11) Kumar, S. V.; Lucyshyn, D. Studying transcription factor binding to specific genomic loci by chromatin immunoprecipitation (ChIP). Methods Mol Biol 2017, 1497, 193-203. 